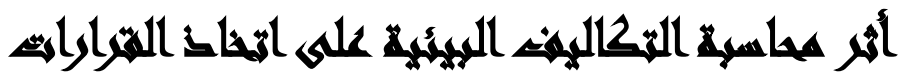

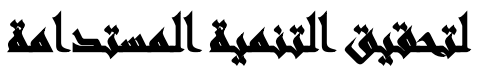

\section{$[r v]$}

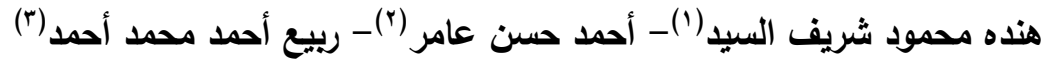

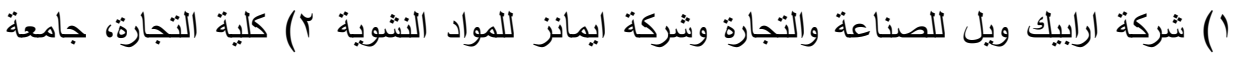

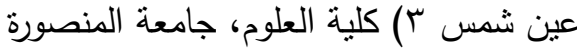

\section{المستخلص}

استهوفت الدراسة إلى بيان أثز محاسبة التكاليف البيئية والإفصاح عنها علي اتخاذ

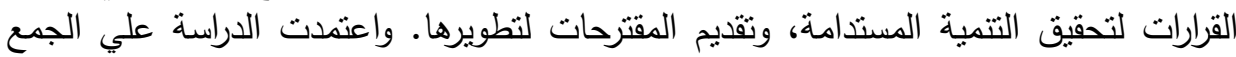

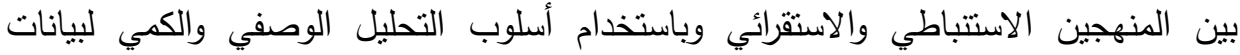

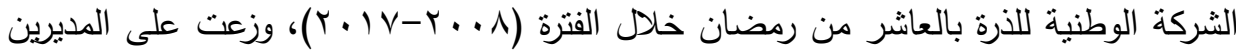

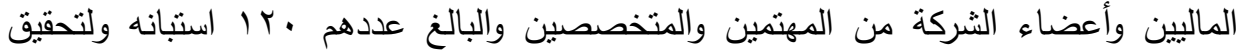

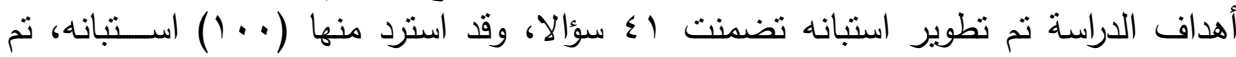

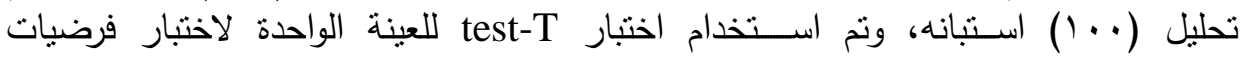

ولقد تبين للباحثين وجود علاقة ذات دلالة إحصائية بين التكاليف البيئية والإفصاح عنها

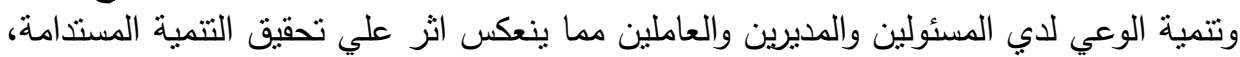

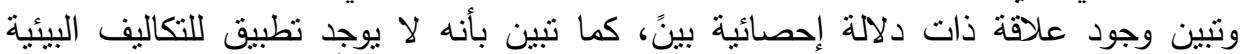

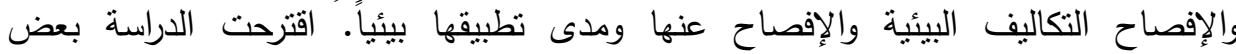

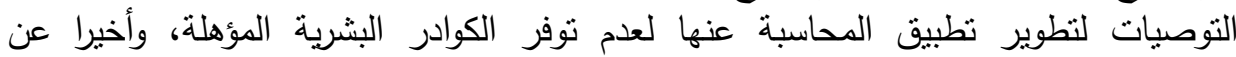

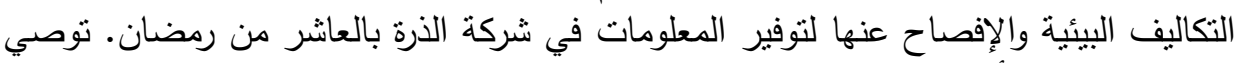

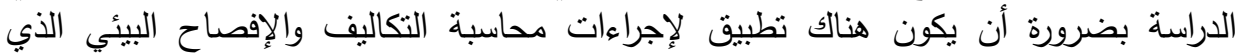

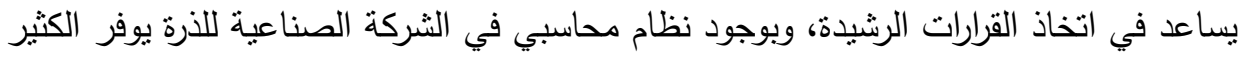

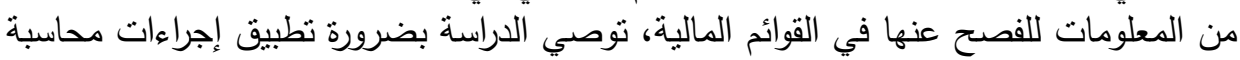

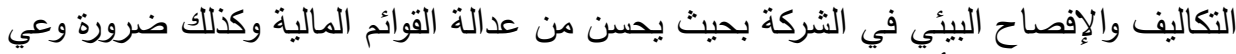

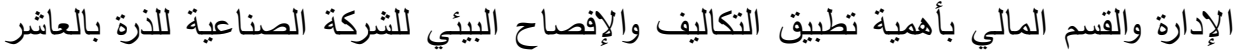

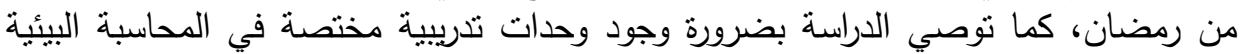

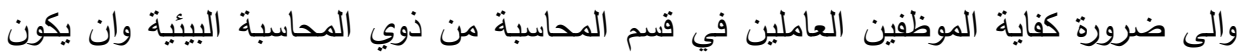
هؤلاء الموظفين على معرفة تامة بالمحاسبة البيئية. الكلمات المفتاحية: التكاليف البيئية، الإفصاح المحاسبة الئي والبيئي، التتمية المستدامة.

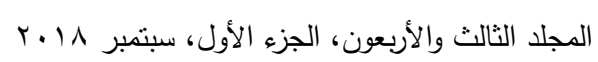




\section{raball}

أصبح موضوع البيئة تزاثا مورثا جيلا بعد جيل حيث لايمكن لأي كائن حي أن يستغنى

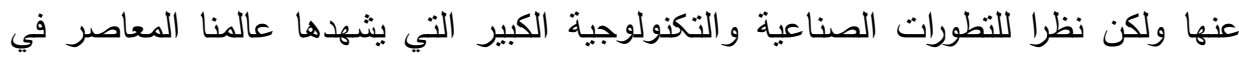
شتى المجالات الاقتصادية والخدمية والذي كان من نتائجه إن أنشئت الصناعات الكبرى ذات الإنتاج الواسع والمنتوع بما يتتاسب مع التقدم الفني والتكنولوجي في وسائل الإنتاج المختلفة مما لإلها أدى إلى الإضرار والتلوث الماء والهواء المحيطين بالإنسان في مصر بعض الفص إن كثيرة المصانع وخاصة في المدن الجديدة مثل (العاثر من رمضان وبدر ومدينة السادات ... وغيرها) مما يؤدى لإنى إلي عواقب بيئيه واجتماعية واقتصاديه واسعة النأثير لا يمكن التنبؤ بها (جهاز شئون البيئة، ودئه

ولذلك إن مصر ليست بمعزل عن العالم الخارجي والمشكلات البيئية تتصف بأنها ظاهرة عابره للحدود أى أنها مشكلة عالمية. وقد بات أمر حماية البيئة والحفاظ عليها وتتميتها امرأ حتميا من قبل الدول في شكل العديد من النشريعات والقوانين، ومن قبل المنظمات المحلية والدولية التي تهنم بالأبعاد البيئية، التي تعمل على منع وتقليل الإضرار البيئية من خلال الأساليب المناسبة لتحقيق الحفاظ على عناصر البيئة المختلفة، بما يحقق التتمية المستدامة. ولذلك لابد من الاهتمام بقضايا البيئة والتكلفة والإيرادات والمنافع المترتبة عنها تتصدر مكانة بارزة وبالغة الأهمية حول العالم بسبب علاقتها الوثيقة برفاهية الإنسان ومستوى معيشته وتقدمه.

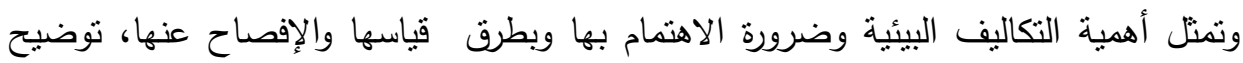
ضرورة الاهنمام بتقويم الأداء البيئي مما يساعد في تحسين ونمو واستمرارية المنشآت الصناعية.

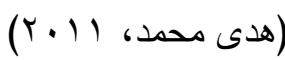

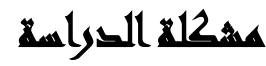

تتبع من فكرة الالتزام البيئي للمنشأة وهو النشاط المولد لواقعة الإنفاق والمتسبب في حدوث عناصر التكاليف البيئية التي تمثل القاسم المشترك بين ذلك المستحدثات في المنظمات التي

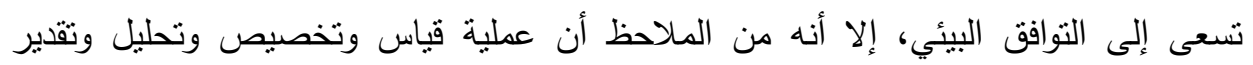
التكاليف البيئة لم يصل بعد إلى المستوى المرغوب فيه، ويرجع ذلك إلى العديد من العوامل التي 
منها عدم وجود مفهوم محدد للتكاليف البيئية من وجهة نظر بعض المنشات الصناعية. ولذلك اعتادت غالبية المنشات الصناعية على إدراج تلك التكاليف ضمن عناصر التكاليف غير المباشرة، مما يترتب عليه أنه لا يمكن تخصيص والإفصاح عن التكاليف البيئية بشكل سليم إليه

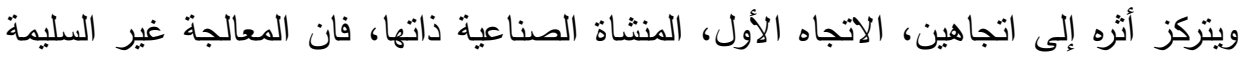
للتكاليف البيئية نوثر على مصداقية القوائم المالية، من حيث عدم توافر المعلومات المحاسبية التي أكدت عليه المعايير المحاسبية المصرية والدولية، وبما ينعكس أثره سلبا على مستخدمي توني تللك القوائم ـ كنلك فإن عدم الإفصاح عن التكاليف البيئية في منن القوائم المالية يفقد المنظمة القدرة على تقدير تلك التكاليف بشكل موضوعي، كما إن عدم التقدير الموضوعي للتكاليف البيئية يؤثر على جودة عمليات التخطيط، والرقابة، واتخاذ القرارات الإدارية، ذات البعد البيئي.

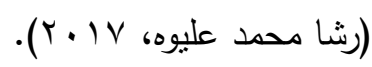

أما الاتجاه الثانى فيرتبط بالحفاظ على البيئة، حيث إن المعالجة الموضوعية للتكاليف

البيئية سوف توثر ايجابيا على عناصر البيئة، وذلك من خلال خفض مصادر التلوث وترشيد استخدام الموارد الطبيعية، بما يحقق التتمية المستدامة، وعدم الجور على حقوق الأجيال القادمة.

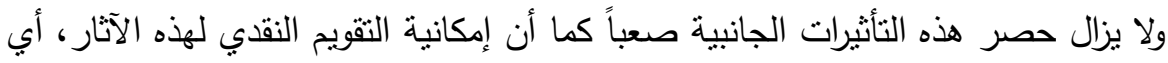
حساب التكاليف البيئية، والفرق بين التكاليف الإجمالية (التكاليف الخاصة + التكاليف الاجتماعية) وبين التكاليف الخاصة. وهذه التكاليف البيئية ما هي في الحقيقة إلا تكاليف

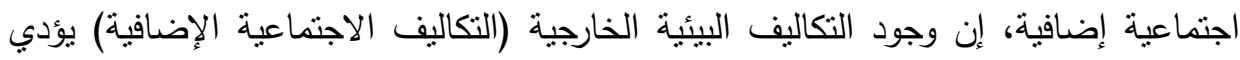
إلى الأضرار بالاقتصاد والبيئة الحالية إن الاستخدام الإنتاجي للبيئة سوف يصبح أكثر تكلفة وأعلى ثمناً نظراً لأن ذلك يتطلب تتقية المياه الملوثة وتصفية الهواء المحمّل بالأكسيد وتحسين الإنه التربة المجهدة والوقاية من الضوضاء. غالباً ما نكون السلع التي تؤدي إلى تخريب في في البيئة

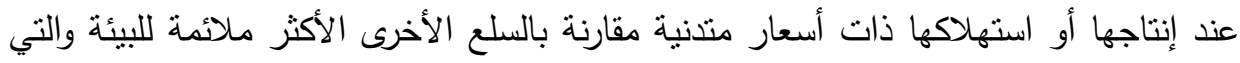

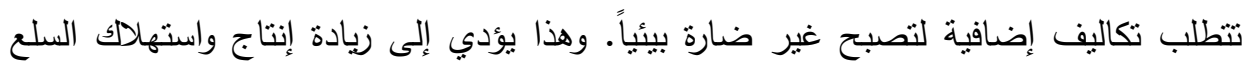

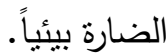


هناك اتجاه أن يلتزم المحاسب بوجهة النظر الاجتماعية، أي أن التقارير المحاسبية يجب

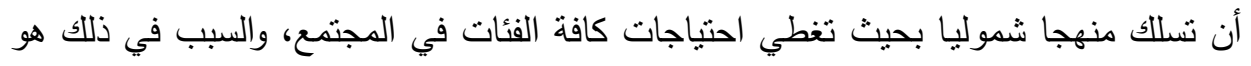

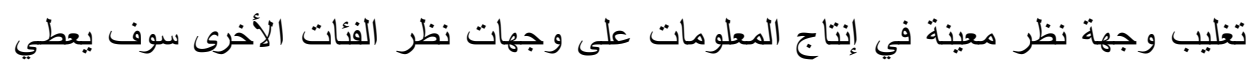
هذه الفئة ميزة نسبية الأمر الذي يؤثنر على عدالة توزيع المنافع فيم بين الفئات المكونة

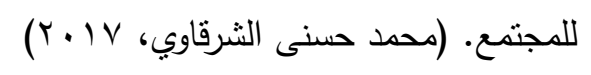

\section{أسهئلا الهمهن}

"ما مدي وجود صعوية في فصل التكاليف البيئية عن الاقتصادية لتحقيق التنمية المستدامة؟"

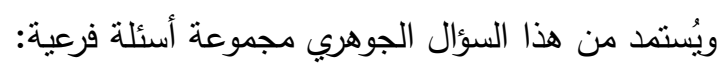

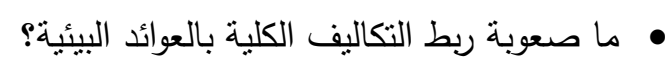

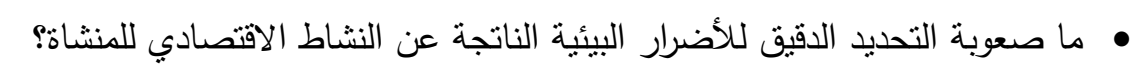

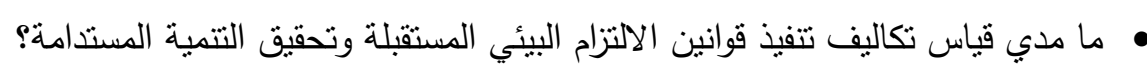

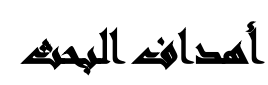

يتمثل الهدف الرئيسي للاراسة في دراسة أثز محاسبة التكاليف البيئية على اتخاذ القرارات لتحقيق التتمية المستدامة. ويتم تحقيق هذا الهدف من خلال مجموعة من الأهداف الفرعية التالية:

ا ب البيان مدى الحاجة إلى محاسبة التكاليف البيئية. r. دراسة أساليب قياس وتحليل عناصر التكاليف البيئية. وبيان دور أساليب الإنتاج المتطورة في ظل وجود التكاليف البيئية. r. التعرف علي المحاسبة البيئية المتعلقة بحماية البيئة والالتزام بكفاءة التكاليف مع القوانين البيئية والتعليمات الدائمة المطبقة في السياسات البيئية.

\section{أهمية المهيث}

• تتبع أهمية البحث من الأهمية الاجتماعية للمحاسبة ومساهنها في التحسين البيئي،

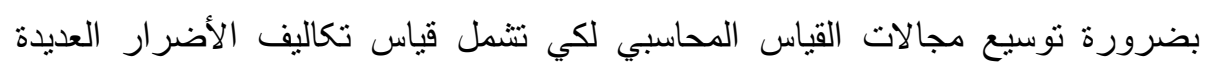


الناتجة عن مختلف أنواع التلوث التي تتسبب فيها المشروعات الصناعية والتي تلحق بتلك المشروعات والعاملين فيها والمجتمعات المحيطة بها، و أيضا قياس تكاليف عمليات

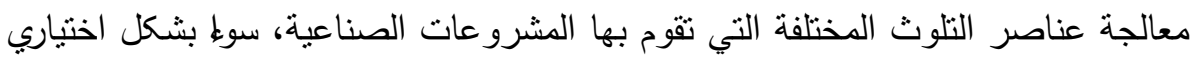

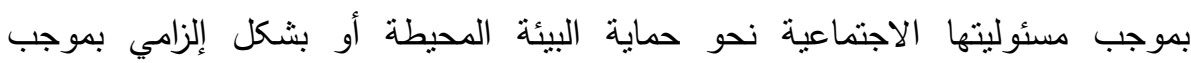

$$
\text { التشريعات البيئية. }
$$

هالاهتمام بالدور الذي تلعبه المحاسبة في قياس وتحليل وتقييم المحافظة على البيئية والإفصاح عن المعلومات البيئة بما يساعد المنشآت على التحسين والتطوير المستمر ودعم

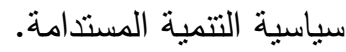
• الاهتمام بضرورة مساهمة المنظمات المحاسبية في مصر لأهيه الدراسات الدحاسبية المتعلقة بقياس تكاليف أضرار التلوث وتكاليف معالجته، وبيان المزايا التي يمكن أن لهمبه تعود على المشروعات الصناعية والمجتمع من وراء إجراء منل هذه الدراسات، عن طريق عقد الندوات و المؤتمرات العلمية وتتجيع البحوث المحاسبية في هذا المجال. • توفير دليل حول اتفاق أو اختلاف بيئة الأعمال المصرية مع بيئات الإعمال الاجنبيه من فن فئن حيث العوامل المؤثرة على مستوي الإفصاح البيئي في التقارير المالية السنوية. توفير قاعدة معلومات مناسبة للجهات التي تهنم بالإفصاح البيئي في مصر حول العوامل المؤثرة على مستوى الإفصاح البيئي بما يمكنها من اتخاذ القرارات والتدابير الملائمة لزيادة مستوي الإفصاح البيئي والتشجيع عليه.

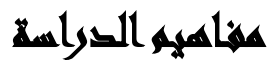

المحاسبة البيئية: نظام وعلم اجنماعي يقود إلى فهم وإدارة أفضل الجوانب البيئية وما يتعلق بها من تكاليف، وتعمل على تحديد وقياس نقدي لقيمة الأضرار البيئية التي تسبيها مؤسسة

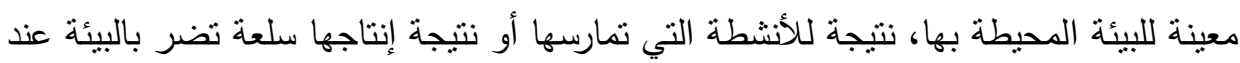

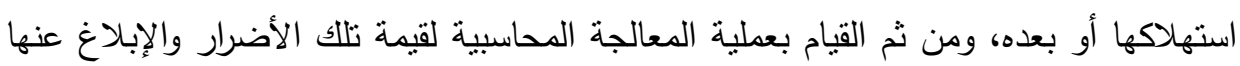
في القوائم المالية سواء للتكاليف الملموسة أو غير الملموسة، وقد يشمل التوسع بعمل تقرير بيئي

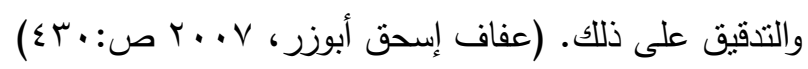


أيضا إن مفهوم التكاليف يعد من المفاهيم المحاسبية والاقتصادية، وتعني التكاليف بشكل

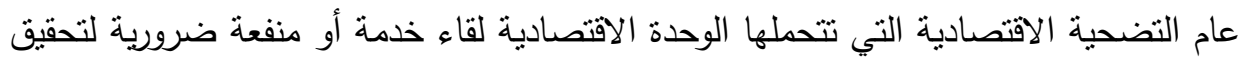

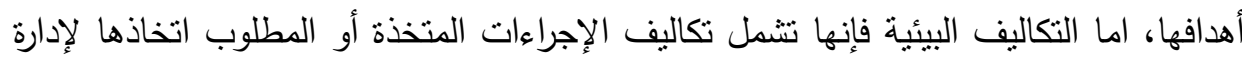

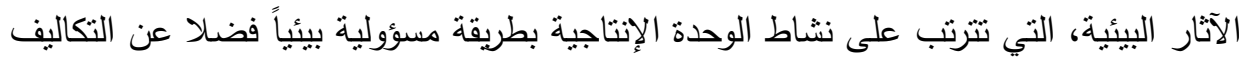

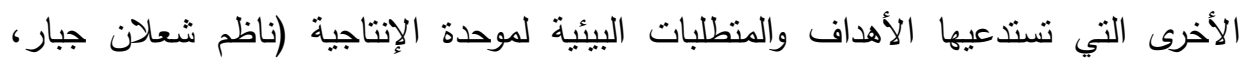
. (0.-V0, T. . . O

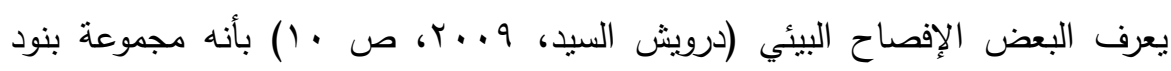

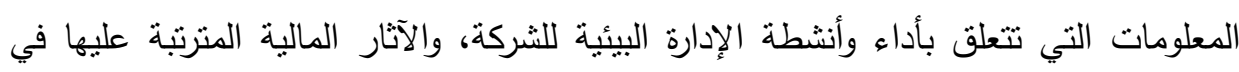

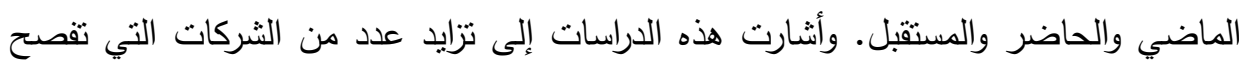
عن المعلومات البيئية في تقاريرها المالية السنوية؛ لتحقيق رغبات المستثرين وباقي الأطراف

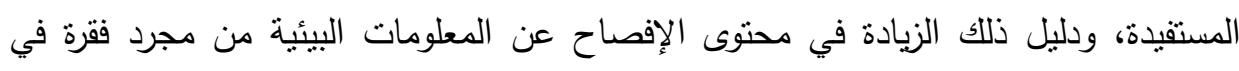

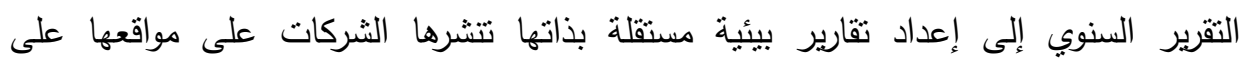
الإنترنت، أو في شكل ورقي مطبوع، ويأخذ هذا الإفصاح العديد من الأشكال كالبيانات الوصفية، الوقائع الكمية والأرقام والملاحظات بالقوائم المالية.

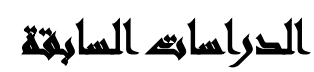

تتاولت العديد من الدراسات السابقة العلاقة بين مستوى الإفصاح البيئي عن المعلومات

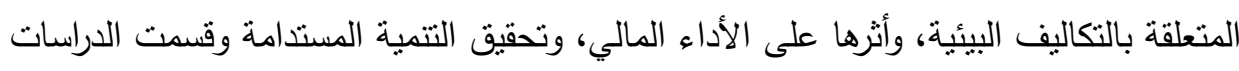
السابقة الى ثلاثة مجموعات:

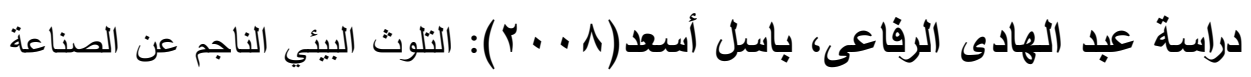
الثقيلة إمكانية قياسه محاسبيا - دارسة تطبيقية عملية علي شركة مصفاة بانياس لتكرير النفط. الهرف: حصر التكاليف التي تتحملها هذه المنشات في حسابات خاصة. وتحسين الأداء البيئي للمشرعات المختلفة. وتحقيق المميزة النتافسية بين الثركات.

المتغيرات: الآثار البيئية للصناعات الملوثة - القياس المحاسبي - الاهنمام بقضايا البيئة تحقيق التنمية المستدامة. 
الفروض: هناك علاقة طردية بين تقييم الآثار البيئية والقياس المحاسبي. هناك علاقة طردية بين الاهنمام بقضايا البيئة وتحقيق التنمية المستدامة. الاستنتاجات والتوصيات: يمكن تلخيص أهم النتائج التي تم التوصل إليها من خلال هذا البحث

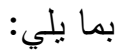
• تلعب المحاسبة دورا في حماية البيئة والحد من التلوث، من خلال معايير وافصاحات خاصة، تجسد مفهوم المحاسبة البيئية.

إن الدور الذي تلعبه المحاسبة البيئية في الحد من التلوث يتمنل في إظهار حجم النفقات التي تتحملها الثركاء جراء قيامها بنشاطات وقائية أو علاجية لحماية البيئة المختلفة. مإن الإفصاح عن بنود التكاليف البيئية في الثركة أمر ليس صعبا فمعظم هذه التكاليف يسهل قياسها وتقدير قيمتها. • إن إمكانية القياس المحاسبي للنقات البيئية متوفرة ويحتاج الأمر إلى تعديل النظام المحاسبي بما يتوافق مع المعايير والمبادئ الدولية للمحاسبة البيئية. دراسة رانيا كمال عبد الجواد أبو ريا(؟ . . ب): المحاسبة عن الآثار البيئية واتخاذ

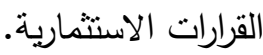
الهرف: يهدف البحث إلى دراسة دور المحاسبة البيئية في ترشيد القرارات الاستثمارية وفحص إمكانية تطوير نظام المعلومات المحاسبية للمحاسبة عن الآثار البيئية للوحدة الاقتصادية:

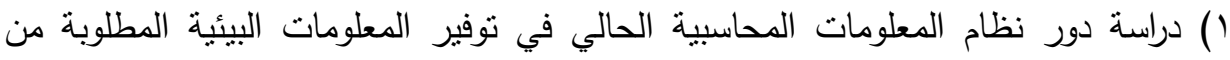
خلال فحص التقرير السنوي لعينة من الثركات التي تتداول أسهمها بالبورصة.

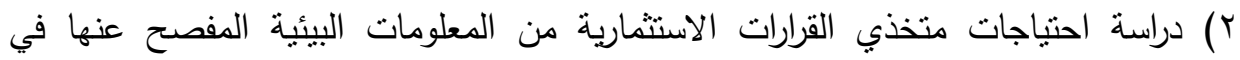
التقارير السنوية للوحدات الاقتصادية وخصائص. النتائج: بالرغم من وجود زيادة عامة في مستوى الإفصاح البيئي للوحدة الاقتصادية إلا أن

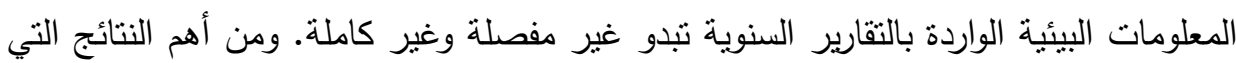
أسفرت عنها الدراسة الحالية هي: 
• عدم كفاية الإفصاح البيئي للوحدة الاقتصادية لأغراض اتخاذ القرارات الاستثمارية، الأهمية

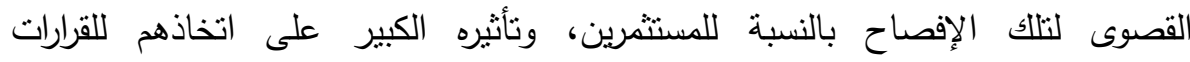

الاستثمارية.

بالإضافة إلى أن الإفصاح البيئي للوحدة الاقتصادية يرتبط موجباً ببعض خصائص المنشأة،

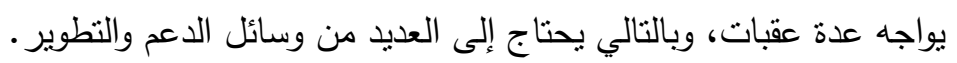

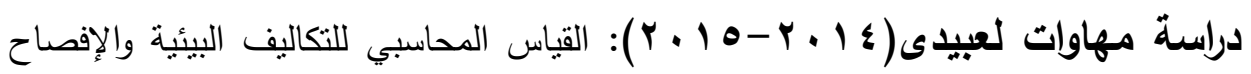

عنها في القوائم المالية لتحسين الأداء - دراسة حالة مجموعة من المؤسسات الصناعية. الهاف: دراسة القياس المحاسبي للتكاليف البيئية والإفصاح عنها في القوائم المالية لتحسين الأديا البيئي وتم التأكد من ذلك من خلال دراسة ميدانية لمجموعة من مؤسسات صناعة

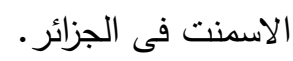

المتغيرات: التلوث - التشريعات البيئية - المسؤولية الاجتماعية - الموارد الاقتصادية - التتمية

المستدامة - حماية البيئة - حقوق الإنسان.

الفروض:

• أهمية فرض التشريعات البيئية وتطبيقها يودى إلى الحد من التلوث الناتج من هذه

• • حماية البيئية تؤدى إلى التنمية المستدامة.

• تطبيق مبدأ الحفاظ على حقوق الإنسان يؤدى إلى زيادة الموارد الاقتصادية.

النتائج:

• أدى النمو الاقتصادي إلى تحقيق أرباح معتبرة، ولكن في نفس الوقت أدى إلى إحداث

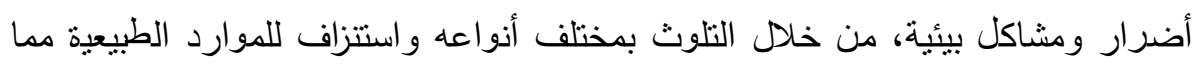
يكون له تأثثر سلبي فى الرفاهية الاجتماعية للأفراد و على التتمية المستدامة.

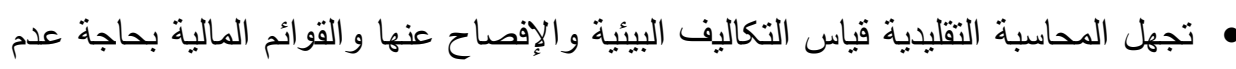
توفر أدوات ووسائل قياس مثل هذه التكاليف ولد الحاجة إلى ضرورة تضمين نالك القوائم

بالبيانات الخاصة بالأنشطة البيئية. 


\section{دراسة هوانغ كونغ( ع 1 ـ Y): تطوير وتتفيذ المحاسبة البيئية في الصين.}

الهدف: تهدف للحد من المشكلة التي تسببها الثغرات معايير محاسبية مختلفة. حاليا، قد

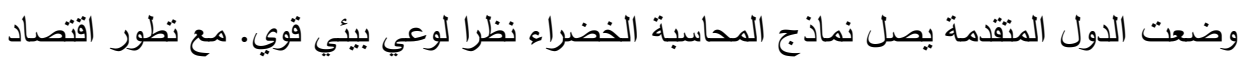

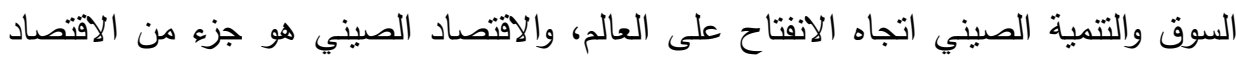

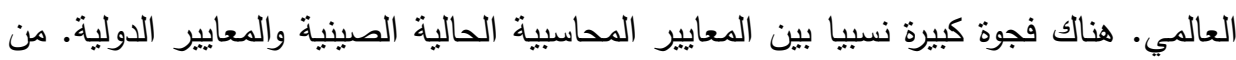
المهم جدا بالنسبة لنا أن ندرك أهمية التكامل مع معايير المحاسبة الدولية. على طريق الإصلاح والمحاسبة، والاستفادة من التجارب المتقدمة السابقة في الخارج. بناء معايير المحاسبة الخضراء مناسبة ونماذج لثركات الصينية. وتكرس لاستكمال التكامل مع الدحاسبة الدولية.

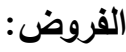
• يوجد علاقة طردية بين المحاسبة البيئية والحفاظ على الموارد الطبيعية. • يوجد علاقة طردية بين تطوير المحاسبة البيئية والحد من النلوث.

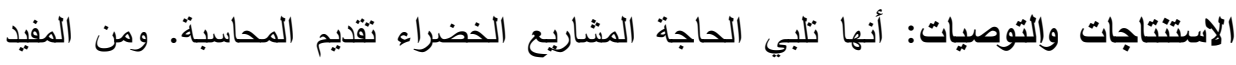

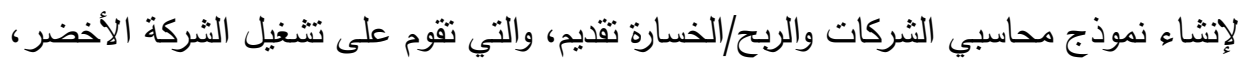

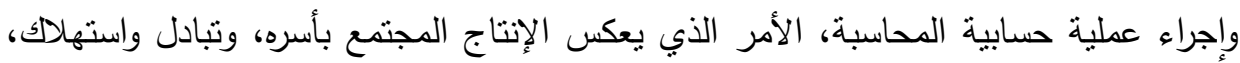

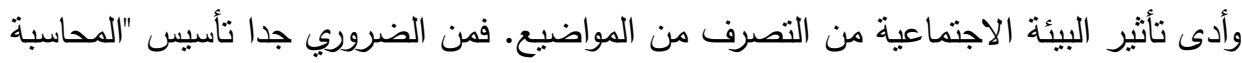

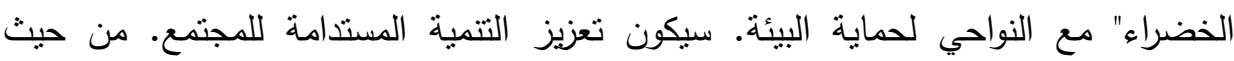
المحاسبة والمحاسبة الخضراء مثل هذا اتجاه التتمية من الآن فصاعدا. دارسة هدى سراج الاين محمد(11 ـ ب): التكاليف البيئية ودورها في تقويم الأداء البيئي في المنشآت الصناعية. الهدف: توضيح مدى أهمية التكاليف البيئية وضرورة الاهنمام بها وبطرق قياسها والإفصاح

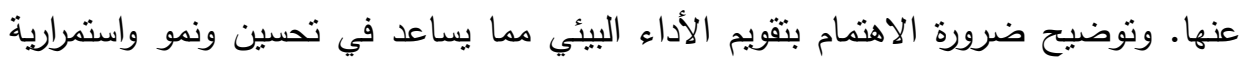
المنشآت الصناعية. • أن نظم المحاسبة التقليدية الحالية لا تقبس التكاليف والمنافع البيئية بشكل ملائم وعادل.

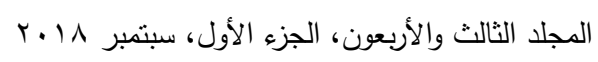


• أن غياب الإفصاح المحاسبي عن الأداء البيئي في القوائم المالية للثركة يؤدي إلى غياب

$$
\text { الإدراك البيئي لاى الأطراف المتيمة. }
$$

• أن إفصاح الثركة عن الأداء البيئي الإيجابي وتوضيح مدى اهتمام الثركة بالجوانب البيئية

$$
\text { التوصيات: }
$$

• إصدار تشريعات تلزم المنشآت بعرض التكاليف البيئية في القوائم المالية.

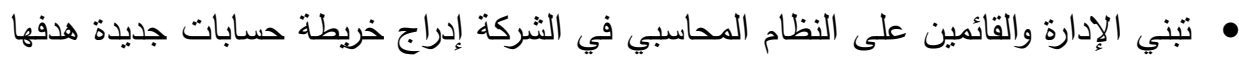

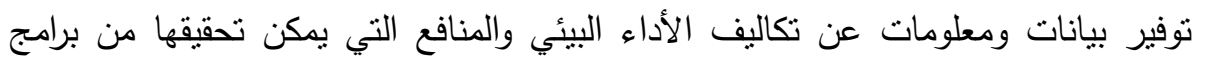

$$
\text { تحسين الأداء البيئي وتطويره للشركة. }
$$

\section{دراسة عمر إقبال توفيق المشهداني؛ علي خلف الركابي(11 + إ): دور المحاسبة}

في المحافظة على البيئة ودعم التتمية المستدامة.

• • توضيح وتقييم دور المحاسبة في تحقيق التتمية المستدامة.

• بيان مساهمة المحاسبة والنظام المحاسبي في المحافظة على البيئة ودعم التتمية المستدامة.

$$
\text { • • إيجاد صيغة مفاهيمه ترتكز على التتمية المستدامة. }
$$

المتغيرات: الحفاظ على البيئة - الموارد الطبيعية - التتمية المستدامة - صحة القوائم المالية.

• يوجد علاقة طردية بين الحفاظ على البيئة والموارد الطبيعية. • • بوجد علاقة طردية بين صحة القوائم المالية والتتمية المستدامة.

الاستنتاجات:

• لا يزال هنالك قصور في إيجاد الصيغ المحاسبية التي تربط بين الأبعاد الثلاثة للتنمية المستدامة بآن واحد. • التتمية المستدامة مفهوم معقد يفتح الباب للعديد من التفسيرات والمواضيع الخاصة بمدى فائدة ومصداقية محاسبة التتمية المستدامة. 
• أن النمو والازدهار الصناعي المتسرع كان له آثار سلبيّة على استتزاف الموارد سواء الناضبة منها أو المتجددة، ولم نساعد النظم المحاسبية القومية على مواجهة القضايا البيئية

$$
\text { والتتمية. }
$$

Che-Ahmed, Ayoib; Osazuwa, Nosakhare Peter; Mgbame, دراسة

Chijoke Oscar

الهدف: التعرف على أثز المحاسبة البيئية على الأداء المالي للثركات في نيجيريا. فإنه يستخدم

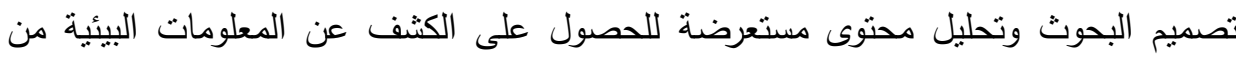
التقارير السنوية المدققة. تحليل الانحدار، واعتماد طريقة المربعات الصغرى العادية، يتم استخدام وتكثف النتائج أن هناك علاقة ذات دلالة إحصائية بين الإفصاح المحاسبي البيئي

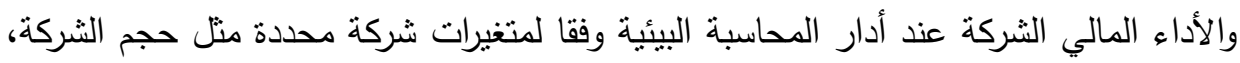
نوع الصناعة وشركة مدقق حسابات.

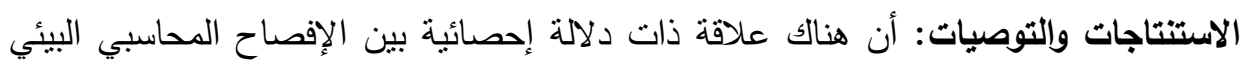

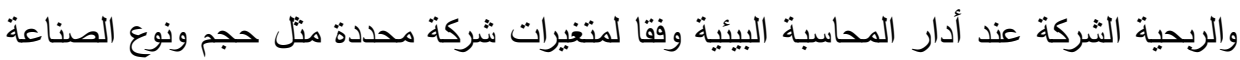

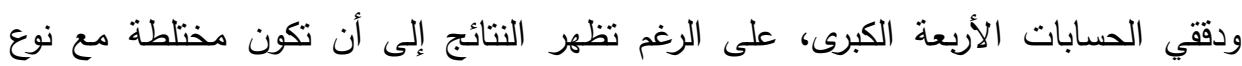
الصناعة ومدققي الحسابات الأربعة الكبرى تظهر علاقة إيجابية. دراسة عبد الله بن جمعان الغامدي(Y V . P): التتمية المستدامة بين الحق في استغلال الموارد الطبيعية والمسئولية عن حماية البيئة. الهدف: الاستعراض النقدي للفهوم الحداثة والنتائج السلبية المترتبة على تنبيه في نظرية التتمية وخاصة في مجال البيئة. التعريف بمفهوم التتمية المستدامة. التعريف بوجهات النظر المختلفة حول الأزمة البيئية وسبل مواجهتها. النتائج • عدم استهلاك الموارد المتجددة بوتيرة أسرع من قدرتها على التجدد أو بطريقة يمكن أن تؤذي

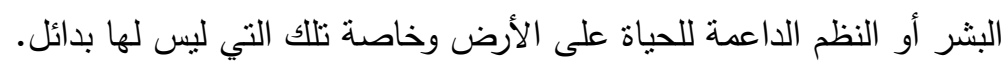


• التوسع في مجال الاعتماد على الطاقة النظيفة المتجددة كالطاقة الثمسية والطاقة المائية وطاقة الرياح. واستخدام الفضلات التقليدية كموارد قدر الإمكان مع التخلص منها عند

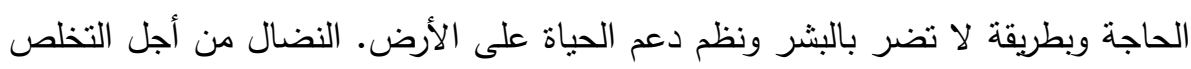

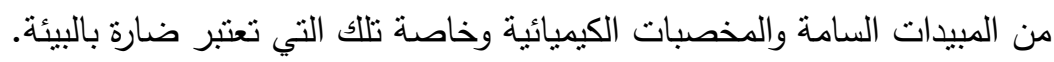
التوصيات والاقتراحات: فيما يتعلق بحماية البيئة والعمل على استدامتها استهلالك الموارد باعتدال

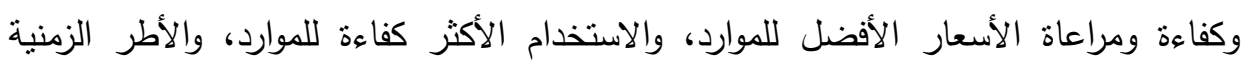
لاستبدال الموارد غير المتجددة بموارد بديلة، والاستخدامات البديلة المحتملة للموارد.

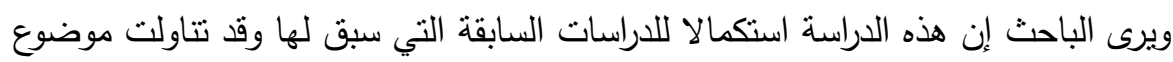

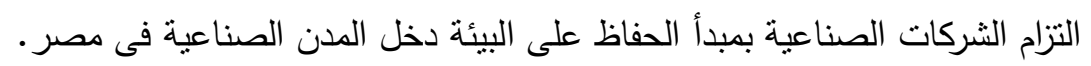

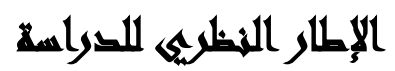

مفهوم وأنواع محاسبة التكاليف البيئية: تعد مناهج المحاسبة البيئية النهج المادي مقابل النهج النقدي يتم اعتماد تهجين في المحاسبة البيئية. أولاً، اقترح المنهج الفيزيائي من قبل الأمم

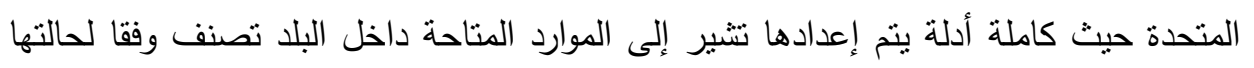

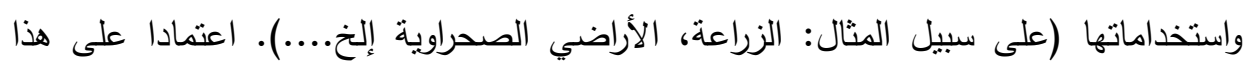

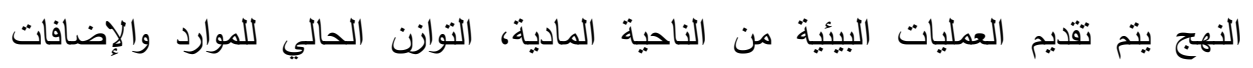
والخصومات من هذا المورد.

وترى الباحثة إن المحاسبة البيئية هي الحفاظ علي وجود علاقة بين الثركات الصناعية

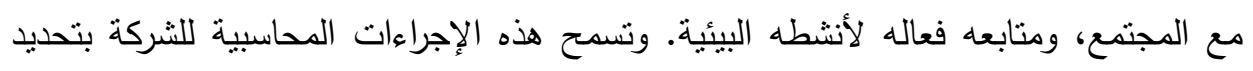

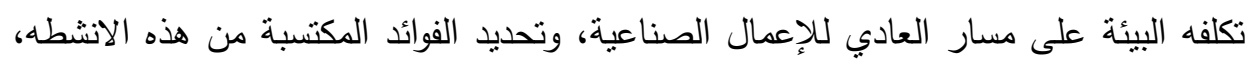

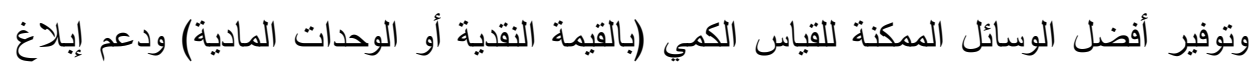
نتائجه.

التكاليف البيئية: توجد عدة تعاريف للتكاليف البيئية منها: التكاليف البيئية هي عبارة عن تضحيات، وقد تكون صريحة أو ضمنية تقوم بها المؤسسة وهذا من أجل الإلتزام بالأداء البيئي

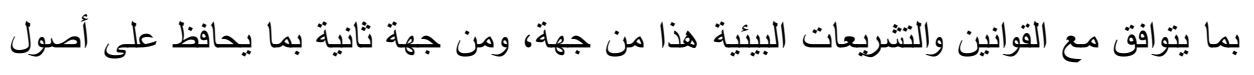


وممتلكات المؤسسة، ومن جهة ثالثة بما يلبي رغبات المستهلكين والأطراف الخارجية الأخرى

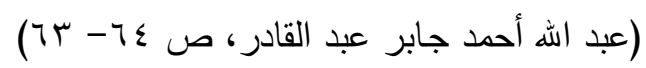
وهناك من يميز بين التكاليف البيئة التي يتحملها المشروع والتي يتحملها المجتمع

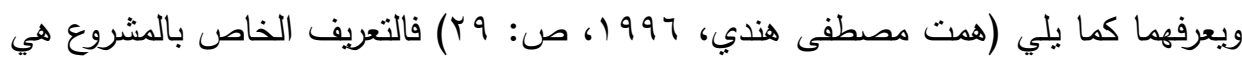
"قيمة ما يتحمله المشروع من أضرار مادية وبشرية أثناء عمليات الإنتاج".

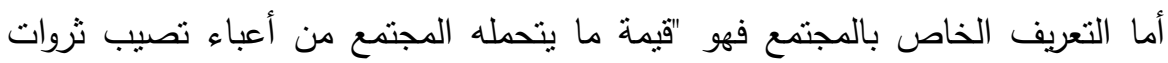
وممتلكات الأطراف الأخرى يكون المشروع قد نسبب فيها". قد قامت إحدى الباحثين التخصصيين في التكاليف البيئية بتعريفها على أنها: "تشتمل على فئل التكاليف الخارجية والداخلية على حد سواء، تكاليف التخطيط والسبطرة، إضافة إلى تلك المتعلقة بمعالجة التأثثرات البيئية والحد منها". من جميع التعاريف السابقة ترى الباحثة أن المحاسبة التكاليف البيئية تمر في ثلاث مراحل هم:

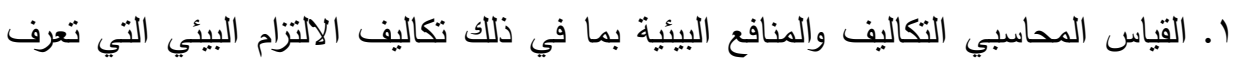
بأنها: "قيمة ما تتعهد تلنزم الثركة بسداده في المستقبل الإصلاح كمعالجة الأضرار البيئية

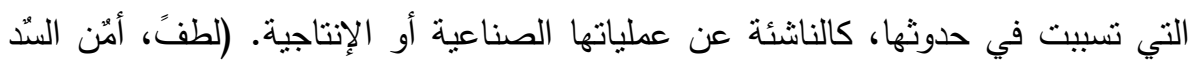

r. الإفصاح المحاسبي عن كل ما يخص التكاليف البيئية كالمنافع والأضرار البيئية. r. اخذ المعلومات البيئية التي نم الإفصاح عنها بعين الاعتبار عند اتخاذ القرارات المتعلقة

$$
\text { بالبيئية. }
$$

وترى الباحثة من خلال ما سبق يمكن تعريف التكاليف البيئية على أنها مجموع ما تتحمله المؤسسة من تكاليف ضمن إطار حماية البيئية بحيث قد تكون صريحة فئنة أو ضمنية ويكون تحملها فى شكل علاج تصحيحي للآثار البيئية أو وقاية من النلوث البيئي أو ضرئية نئية نتيجة الإضرار بالبيئة او منع للتلوث البيئي. أهداف محاسبة التكاليف البيئية: إن تطبيق المحاسبة البيئية لا يعد هدفا فى حد ذاته، وانما

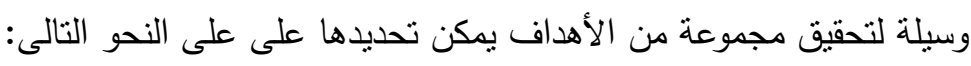


1. المحاسبة البيئية سنساعد على تصريف مسؤولية المنظمة وزيادة بيئتها الثفافية.

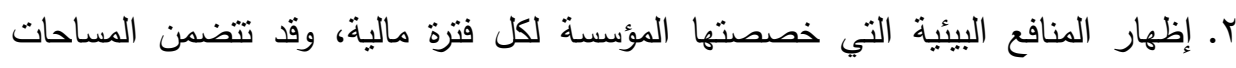
الخضراء المضافة خلال الفترة أو التخفيض في خسائر وأضرار عناصر التلوث البيئي، تخفيض نكلفة العلاج الطبي، أو زيادة إيرادات وأرباح المؤسسة من تطبيق نظام الإدارة

r. يساعد على التفاوض على مفهوم البيئة ويحدد علاقة الثركة بالمجتمع بشكل عام ومجموعة الضغط البيئي على وجه الخصوص. هذا يساعد على منظمة تسعى لإدارة استراتيجيا جديدة

$$
\text { والناشئة قضية مع أصحاب المصلحة. }
$$

ء. بسبب حركة الاستثمار الأخلاقية، والمستثمرين الأخلاقية نطلب من الثركات أن تكون

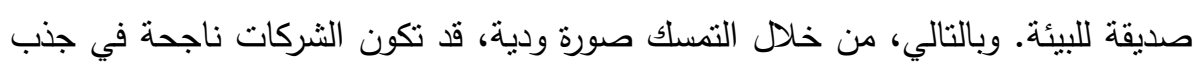

$$
\text { الأموال من الأفراد والجماعات "الخضراء". }
$$

ه. حركة استهلاكيه المحاسبة البيئية التي أطلقتها مجموعات اللوبي البيأية تشجع المستهلكين

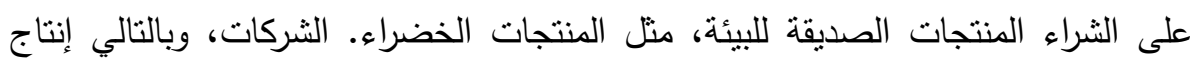
المنتجات الخضراء قد تتخذ ميزة التسويق التنافسية عن طريق الكثف عن نفسه.

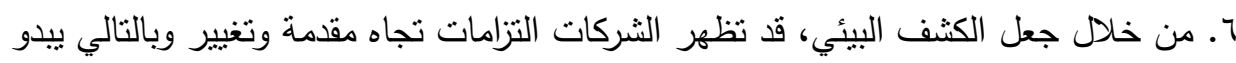
أن تستجيب لعوامل جديدة. V. الشركات العاملة في الصناعات غير الصديقة للبيئة نشأت العاطفة العامة القوية. هناك

$$
\text { لوبي بيئي قوي ضد هذه الصناعات. }
$$

^. يمكن استخدام التقارير الخضراء لمكافحة الجمهور السلبي المحتمل الآراء.

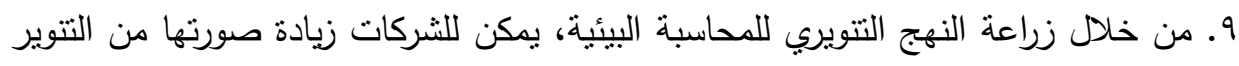

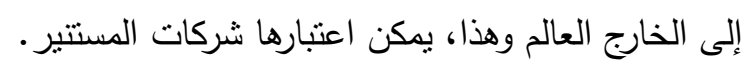

أهمية المحاسبة للتكاليف البيئية: تعد الثركات الصناعية موضوع الدراسة الثركات من القطاعات

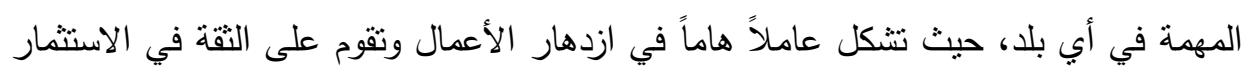
والاقتصاد وذلك من خلال علاقتها الوثيقة مع الهيئات المشروعة والحكومية، ومن خلال تقيدها بالتشريعات والأنظمة المفروضة عليها، لذلك تعد البيانات التكاليف البيئية لشركات الصناعية 
من المصادرة الهامة للمعلومات بالنسبة لمتخذي القرارات الاقتصادية، وسوف يساعد الإفصاح الكافي في هذه البيانات متخذي القرارات الاقتصادية في تقييم المركز المالي والأعمال والإنجازات التي تقوم بها الثركات الصناعية فى الحد من تلوث البيئة. لاثثك ان هناك العديد من الأسباب التى دفعت نحو الاهتمام بالمحاسبة التكاليف البيئية ومنها:

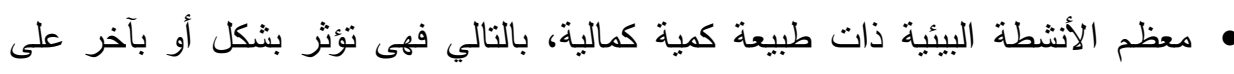

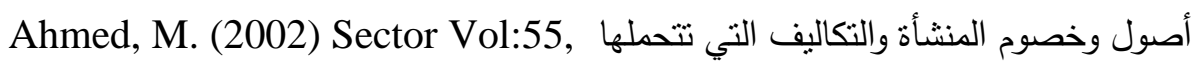

$$
\text { No:30, p 420,421. }
$$

•وسيع نطاق عملية التقييم وتحليل الاستثمار لكي يشمل الآثار البيئية المحتملة. التوصل إلى فهم أفضل للتكاليف البيئية وآداء العمليات والمنتجات وتسعير ها بدقة. • المساعدة في تطوير وتشغيل نظام إداري بيئي للوحدة ككل.

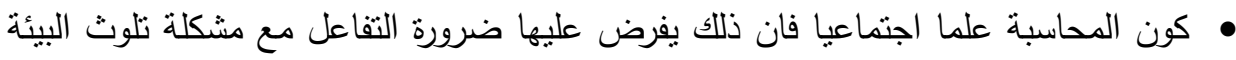
ونفاذ مواردها لان نأخر ها سيؤدي لنتأخر هذا العلم مقارنة بالعلوم الأخرى مثل علم علم الاقتصاد

• تستمد المحاسبة وجودها من اعتراف المجتمع بنتائجها من خلال وظيفتي القياس والتوصيل للمعلومات المالية والاقتصادية للمجتمع واستمرار الطلب على خدمات مهنة المحاسبة والتدقيق مما يستلزم تلبية الاحتياجات المتزايدة للمعلومات البيئية والاجتماعية بجانب

$$
\text { المعلومات المالية. }
$$

• إن تجاهل قياس التكاليف البيئية الناتجة عن تلوث البيئي من شأنه أن بضلل العديد من مؤشرات قياس الأداء ويضخم النتائج.

أثر التكاليف البيئية على الأداء المالي للمؤسسة: لكي تلبي التئي المحاسبة المالية لوظيفتها الأساسية فى إعلام الأطرف الداخلية والخارجية بمحصلة الأداء الاقتصادي والاجتماعي والبيئي

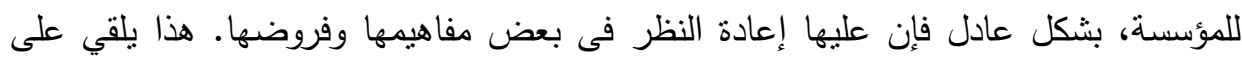

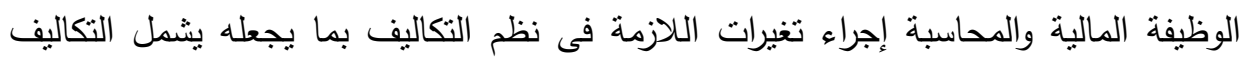

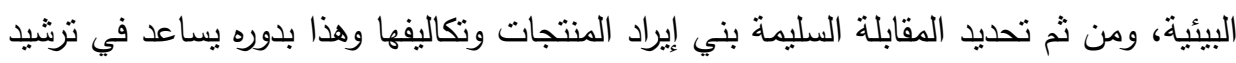

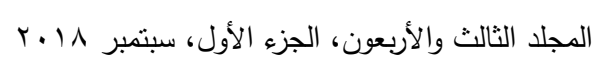


قرارات التسعير، والتي ينتج عنها زيادة أرباح المؤسسة وحصنها السوقية على الددى الطويل والاهتمام بدراسة تكاليف الأداء البيئي، وقياسه يساعد على كيادئ كيفية تحسين الأداء المالي

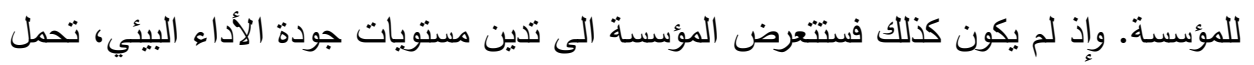
تبعات القانونية فى غرامات والحرمان من حوافز . كما تنظر المحاسبة المالية الى تكاليف البيئة على أنها تمكن المؤسسات من إعداد تقارير لاستخدام المستثمرين والمقترضين من تلكئك التقارير .

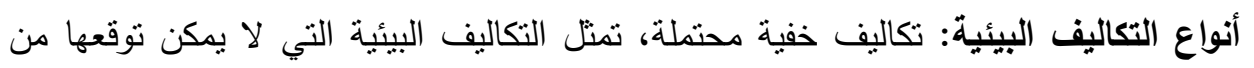

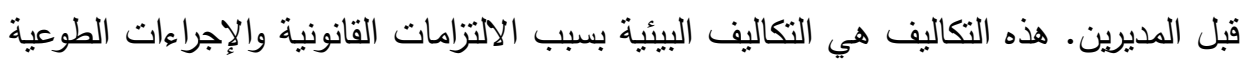
وأنشطة الثركات.

على الرغم من أن هذه التكاليف مصنفة كتكاليف عامة أو تكاليف بحث وتطوير ، قد تكون بعض التكاليف يتم التغاضي عنها قبل العمل كمديري الأعمال التركيز على تكاليف الاستثمارات التي يتعين القيام بها (وكالة حماية البيئة، 990 (1)).

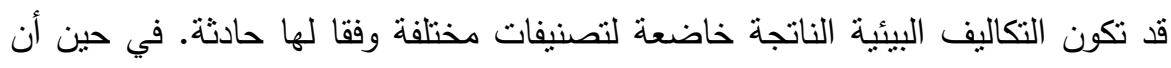
بعض التكاليف البيئية نظهر نتيجة للأنشطة التي نفذت من أجل الغرض من حماية البيئة

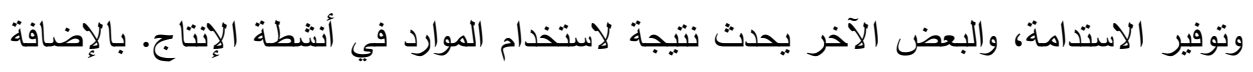

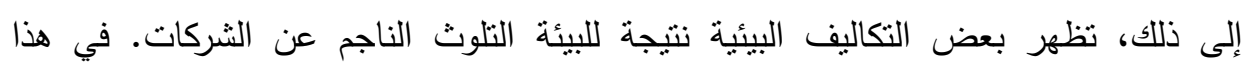
السياق، يمكن فحص التكاليف البيئية تحت ثلاثة عناوين: تكاليف الوقاية؛ وتكاليف التشغيل؛ وتكاليف التتفيذ (Çelik, M. 2008) تكاليف الوقاية: هي التكاليف المتكبدة في عملية التصميم والإنتاج والاستخدام والتدمير لمراحل المنتج من أجل حماية البيئة وتقليل الضرر البيئي. التكاليف المتكبدة في دورة حياة المنتج ؛ تكاليف التخطيط البيئي؛ تكاليف التتسيق البيئي في تصميم المنتج؛ تكاليف إعادة التدوير؛ تكاليف التغليف الصديقة للبيئة؛ نكاليف الإدارة البيئية، ومراقبة النفايات، والتخلص من أو تكاليف العلاج داخل نطاق تكاليف الوقاية. هذه التكاليف هي التكاليف التي يمكن تحديدها وفقا لقرارات الإدارة. تكاليف التشغيل: يمكن تعريفها على أنه سعر الموارد الطبيعية العامة المستقيدة أثناء تشغيل

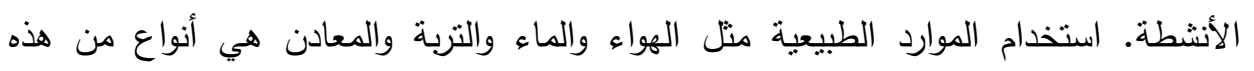


التكاليف. التكاليف المنأثرة هي التكاليف المتعلقة بالأضرار البيئية الناجمة عن فثل المراحل السابقة. يتم تعريف عناصر التكلفة هذه على أنها انخفاض في الحيوانات والنباتات والخسائر التي تسببها النفايات التي يتم إطلاقها في الهواء والماء والتربة.

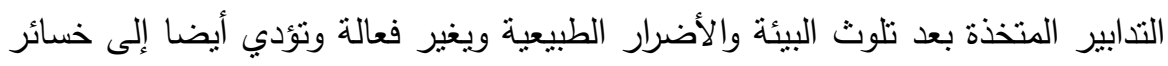

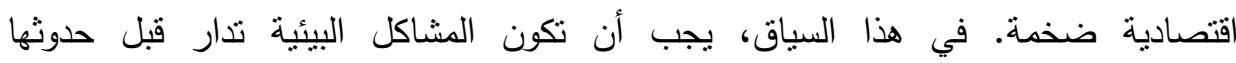

.(Kırımhan, S. 2005)

الأنشطة المنجزة لتقليل المخاطر البيئية التي يحتمل أن تتشأ نتيجة للأنشطة التشغيلية وحماية البيئة والتكاليف المرنبطة بها بمكن أن تؤدي إلى تغييرات هيكلية ومالية على الأعمال.

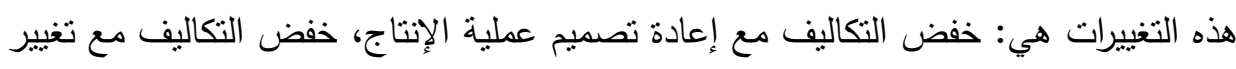

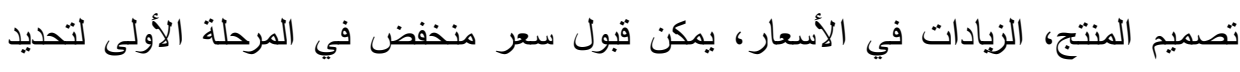

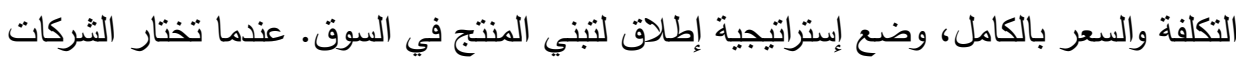
اختبار التأثثر البيئي على شراء الأصول، كيف حساب فروق التكلفة الناشئة التي تغطيها المحاسبة البيئية. في هذا السياق، فإن التكاليف المتكبدة فيما يتعلق بالبيئة ويحسب بالئي بالتفصيل

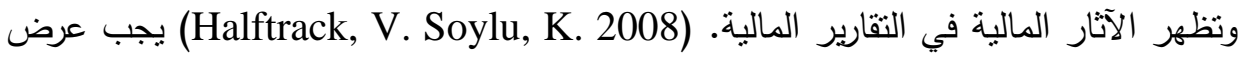
المخاطر البيئية غير المالية بالتقصيل في تقارير التشغيل/البيئة وفي ملاحظات التقرير المالي.

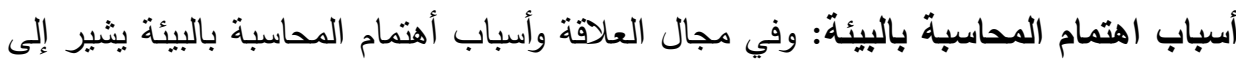

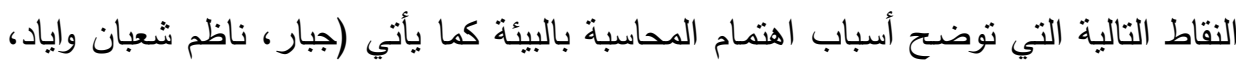

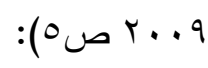
• نظام المحاسبة البيئية يعد أداة لقياس ورقابة واتخاذ القرارات اللازمة لتحقيق النطور •

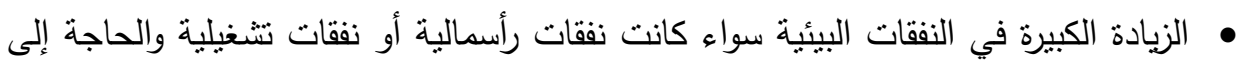

$$
\text { وضع أولويات لهذه النفقات. }
$$

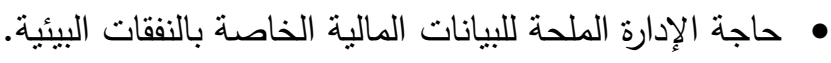
• تبرز الحاجة للمحاسبة البيئية لقيادة التكاليف البيئية (كموجه كلفة). 
هؤدي الاهتمام بالمحاسبة البيئية إلى الوفر في التكاليف لأن التكاليف البيئية عادةً ما تكون جزء من المصاريف الصناعية الإضافية. • الحاجة المتزايدة للبيانات المالية الخاصة بالأداء البيئي من قبل مختلف الجهات كالحكومة، المستثمرين، المقرضين البنوك والمنظمات غير الحكومية لأن عدم نوفر مثل هذه البيانات يساعد بعض المنظمات غير الملتزمة على نلوث البيئة وإتلاف الموارد الطبيعية.

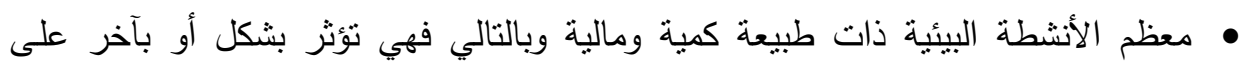
أحوال وخصوم المنظمة والتكاليف التي تتحملها. هنالك حاجة ملحة لأسلوب ملائم لتخصيص وتحميل التكاليف البيئية والتمييز بينها وبين

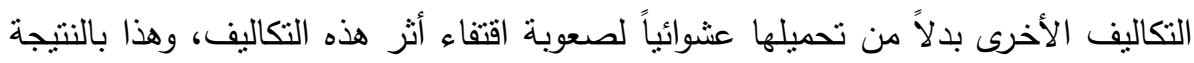
يؤدي إلى أيجاد أسلوب ملائم لتخفيض التكاليف ومن ثم تحديد أسعار المنتجات. تعريف الإفصاح المحاسبي البيئي: يعتبر الإفصاح من الأركان الرئيسية في الفكر المحاسبي،

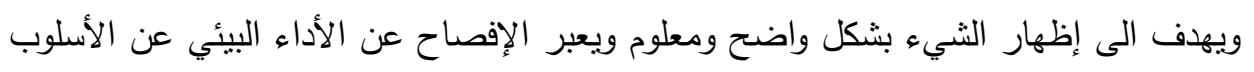

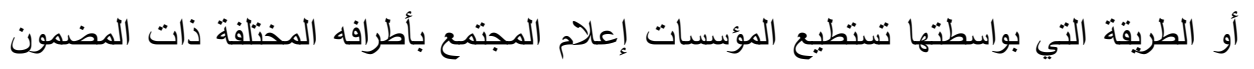

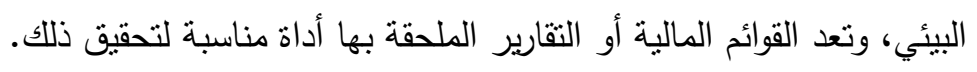
ترى الباحثة أن الإفصاح البيئي يمكن أن يعرف بأنه: "تقديم وعرض البئنة البيانات والمعلومات

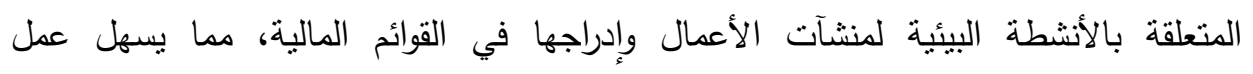
مستخدمي المعلومات ومتخذي القرارات ويؤدي إلى ترشيد القرارات عند تقييم الأداء البيئي لهذه المنشآت. أنواع الإفصاح المحاسبي عن التكاليف البيئية: ويضم الإفصاح المحاسبي البيئي نوعان هما:

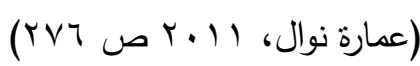
الإفصاح الإلزامي: ظهر مفهوم الإفصاح المحاسبي البيئي الإجباري والإلزامي، وبمقتضاه تلتزم

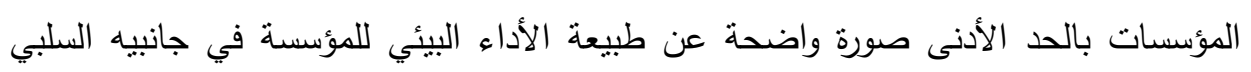

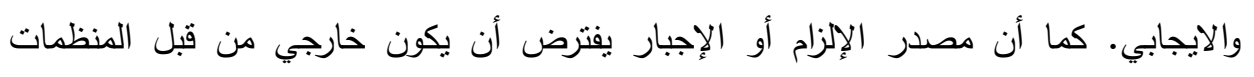
المهنية المحاسبية المحلية أو الدولية، غير أن مصدر الإلو الإلزام يمكن أن يكون من المنظمات المهنية للححاسبة حيث قد تثير القوانين البيئية واللوائح والقرارات المنفذة لها. 
الإِصاح الاختياري: ويتم الإفصاح الاختياري لاستكمال ما يراه المحاسبون ضروريا لتنذية

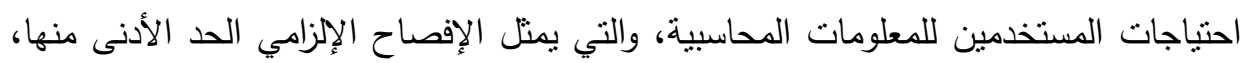

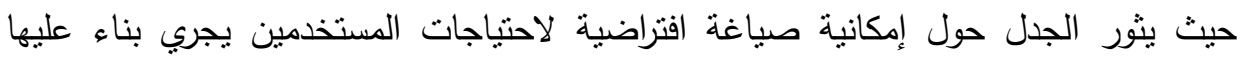

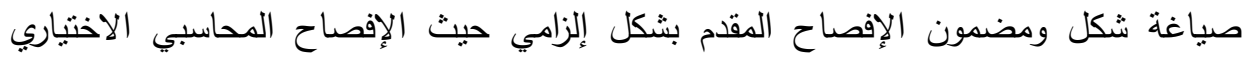
تداركه وتغطينه. ويمكن القول أن الإفصاح البيئي الإختيارى ما هو إلاًّ إستراتيجية نلجأ إليها

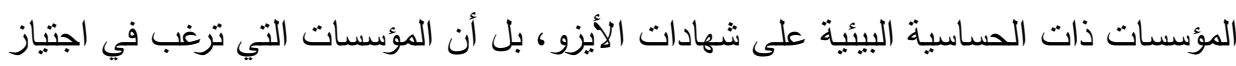
الحدود الجغرافية عليها تبني هذه الإنتراتيجية.

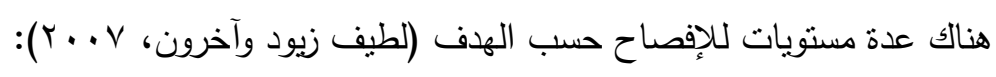

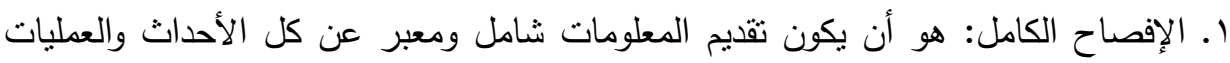

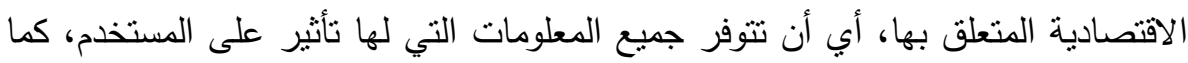

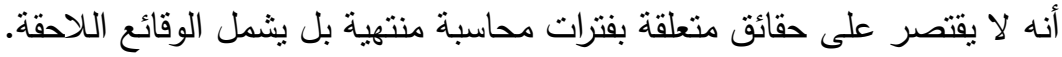

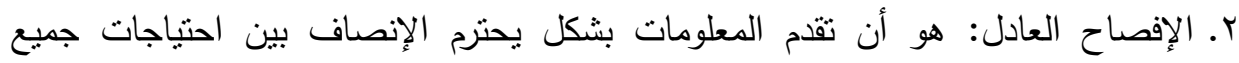

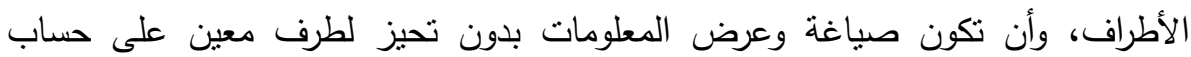

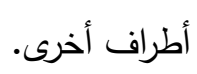

r. الإفصاح الكافي: هو أن تقدم المعلومات بالثكل الذي يراعي الدد الأدنى للمعلومات

$$
\text { الواجب توفيرها في القوائم المالية. }
$$

ع. الإفصاح الملائم: أن تقدم المعلومات في القوائم المالية بلماتية بحيث تكون ذات قيمة ومنفعة بالنسبة لمستخميها وأكثر تتاسبا مع طبيعة النشاط وظروف المؤسسة.

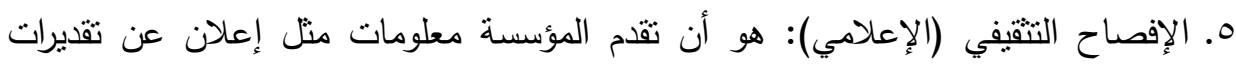

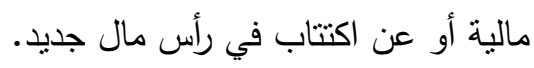

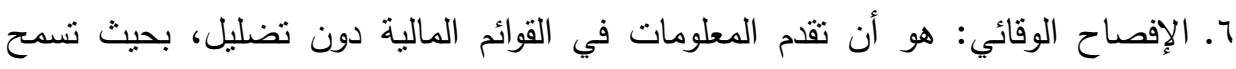
بحماية الأطراف ذوى القدرات المحدودة على الفهم والإدراك للمعلومات الكات المالية الكالية. دور المحاسبة التكاليف البيئية فى ضوء القوانين والتثريعات الدولية: زادت الأحداث البئية البئية

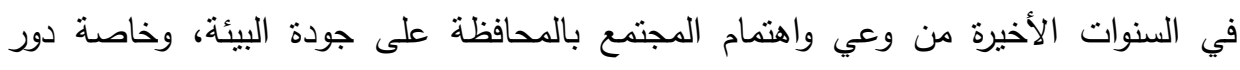

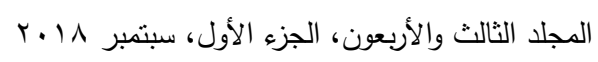


الثركات ومسؤوليتها في هذا الصدد، وأدى ذلك إلى اهتمام المنظمات المهنية والجمعيات العلمية المحاسبية بهذا الموضوع، من خلال العديد من الخطوات والمبادرات التي تشجع الثركات على الاعتراف بالتكاليف البيئية.

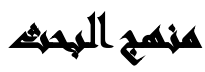

اعتمد الباحثون على كل من المنهج الاستقرائي، والمنهج الاستتباطي، حيث يعتمد على

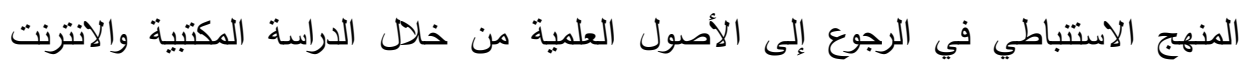

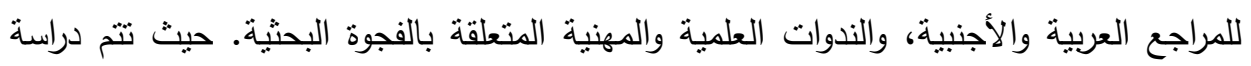
وتحليل ما تتاولته الدراسات السابقة التي وردت في الفكر الدحاسبي فيما يتعلق بمتغيرات الدراسة

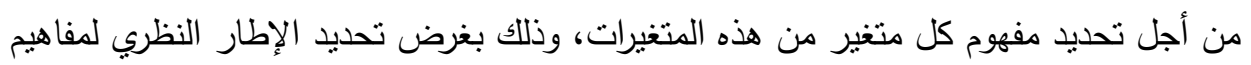

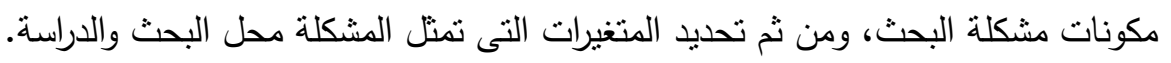

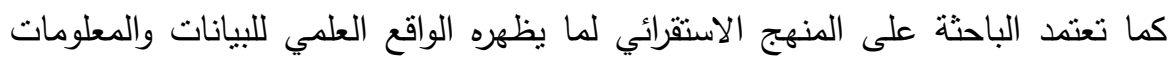

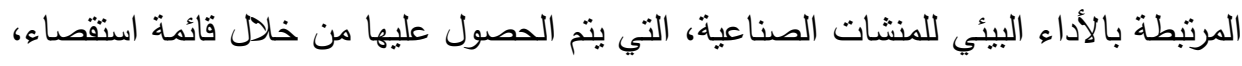

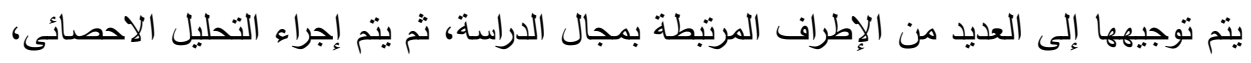
واختبار صحة الفروض البحثية.

\section{أسوائ القراسما}

إعداد قوائم الاستقصاء للتعرف على أثر محاسبة التكاليف البيئية على اتخاذ القرارات

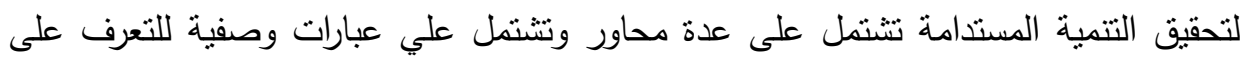
مدى الوعي البيئي. صدق الاتساق الداخلي لفقرات المقياس: يقصد بثبات أداة القياس أن يعطي النتائج نفسها إذا أعبد تطبيق المقياس على نفس العينة في نفس الظروف ويتم قياسه بثلاث طرق: الاختبار: يتم في هذه الطريقة تطبيق المقياس على عينة استطلاعية وتم حساب معامل

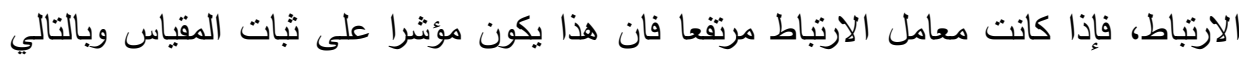
على صلاحية وملائمة هذه المقياس لأغراض الدراسة. 
الثبات عن طريق التجزئة النصفية: حيث يتم تجزئة فقرات المقياس إلى جزأين، الجزء الأول

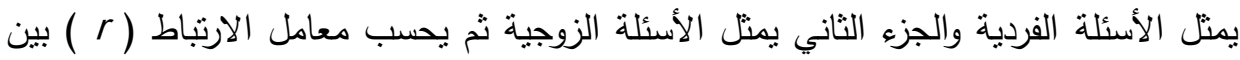
درجات الأسئلة الفردية ودرجات الأسئلة الزوجية ثم تصحيح معامل الارتباط بمعادلة بيرسون

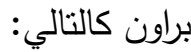
جدول(1): مقياس الثبات (الفا كرنباخ)

\begin{tabular}{|c|c|c|}
\hline قيمة ألفا & عدد العبارات & أبعاد المقياس \\
\hline . . & 1 & المحور الأول \\
\hline.$V 9 V$ & 1. & النحور الثانى \\
\hline.$\vee \vee 0$ & 1. & المحور الثالث \\
\hline . .AMI & 11 & المحور الرابع \\
\hline
\end{tabular}

الأساليب الإحصائية المستخدمة: استخدمت الدراسة أسلوب التحليل الإحصائي الوصفي والكمي

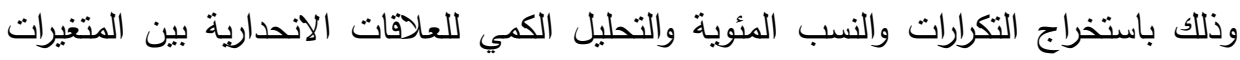
المرتبطة بالدراسة عن التكاليف البيئية والمتغيرات المؤثرة فيها.

\section{ary}

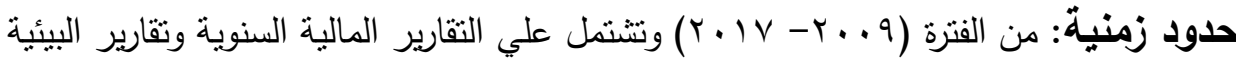

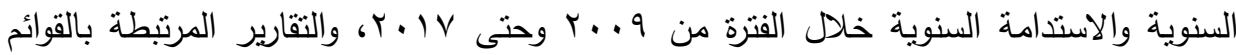
المالية والمتعلقة بمجلس إدارة الثركة الثركة الوطنية لمنتجات الذرة. حدود مكانية: المنشآت الصناعية بالعاثر من رمضان ذات التأثير البيئي السلبي بالتركيز على (الثركة الوطنية لمنتجات الذرة) والمسجلة في سوق الأوراق المالية المصري.

\section{هرون المهند}

الفرض الأول: توجد علاقة ذات دلالة إحصائية بين التكاليف البيئية وتكاليف تشغيل المنشأة. القرض الثاني: توجد علاقة ذات دلالة إحصائية بين التكاليف البيئية ومستوى جودة المنتج

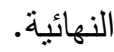


الفرض الثالث: توجد علاقة ذات دلالة إحصائية بين التكاليف البيئية وزيادة ربحية المنشأة. الفرض الرابع: إظهار التكاليف البيئية في القوائم المالية يساعد على سرعة اتخاذ القرارات للمنشأة.

الفرض الخامس: نوافر الإفصاح المحاسبي للتكاليف البيئية يؤدى إلى تحقيق التتمية المستدامة للموارد الطبيعية المتاحة.

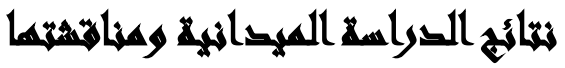

جدول(ץ): الفئات العمرية للعاملين بشركة الذرة بمنطقة العاشر من رمضان

\begin{tabular}{|c|c|c|c|}
\hline$\%$ & التكرار & 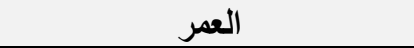 & r \\
\hline $19.1 \mathrm{~V}$ & r & أقل من •r سنة & 1 \\
\hline ro... & $\varepsilon r$ & من · س سنة إلى أقل • ع سنة & r \\
\hline rq.1V & ro & من •ـ سنة إلي أقل من •0 سنة & r \\
\hline $17.7 \mathrm{~V}$ & $r$. & من · 0 سنه فأكثر & $\varepsilon$ \\
\hline $1 \cdots$ & ir. & الإجمالي & \\
\hline
\end{tabular}

المصدر: إعداد الباحثة من بيانات استمارة الاستيان

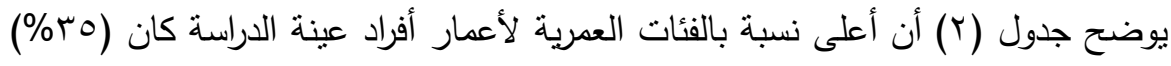

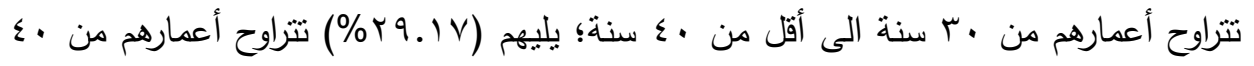

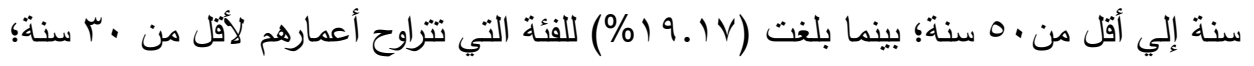

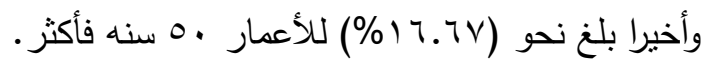

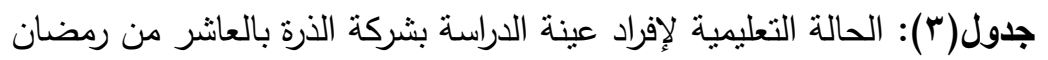

\begin{tabular}{|c|c|c|c|}
\hline$\%$ & التكرار & المؤهل العلمي & r \\
\hline מש.rז & $\varepsilon$. & دبلوم & 1 \\
\hline $19.1 \mathrm{~V}$ & r & ثانوية عامة & $r$ \\
\hline سז.r. & or & بكالوريوس & r \\
\hline r.o. & r & ماجستير & $\varepsilon$ \\
\hline $1.7 \mathrm{~V}$ & r & كتوراة & 0 \\
\hline $1 \ldots$ & Ir. & الإجمالي & \\
\hline
\end{tabular}

المصدر: إعداد الباحثة من بيانات استمارة الاستبيان 


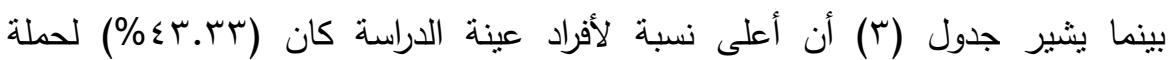
البكالوريوس؛ يليهم (rr.rr\%) لحملة الدبلوم؛ بينما بلغت (19.1\%) لحملة الثانوية العامة؛

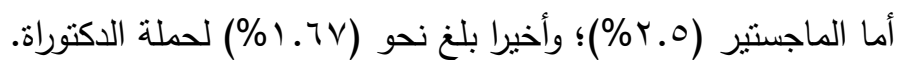

\begin{tabular}{|c|c|c|c|}
\hline$\%$ & التكرار & الوظيفة & 5 \\
\hline $7.7 \mathrm{~V}$ & 1 & مدير عام & 1 \\
\hline $7.7 \mathrm{~V}$ & $\Lambda$ & مدير مالى & $r$ \\
\hline TY.0. & 10 & مدير أداري & $r$ \\
\hline IY.0. & 10 & رئيس قسم & $\varepsilon$ \\
\hline T..AT & TO & محاسب & 0 \\
\hline $17.7 \mathrm{~V}$ & T. & كيميائي & 7 \\
\hline IT.M & 17 & عمالة فنية & V \\
\hline $1 \cdot . \Lambda T$ & $1 \pi$ & عمالة خدمات معاونة & $\Lambda$ \\
\hline $1 \cdots$ & $\pi$. & الإجمالى & \\
\hline
\end{tabular}

المصدر: إعداد الباحثة من بيانات استمارة الاستبيان بينما يشير جدول (ع) أن أعلى نسبة لأفراد عينة الدراسة كان (r^...r\%) لمحاسب؛

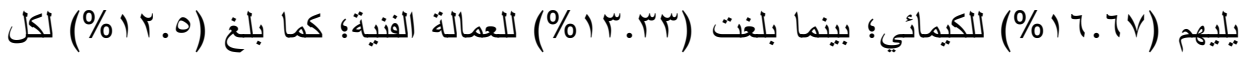
من مدير إداري، ورئيس قسم؛ وبلغ (r^. • (\%) للخدمات المعاونة؛ وأخيرا بلغ نحو (7.7.7\%)

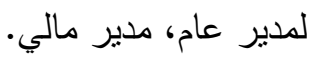
جدول(ه): سنوات الخبرة لإقراد عينة الدراسة بشركة الذرة بالعاشر من رمضان الفيرة

\begin{tabular}{|c|c|c|c|}
\hline$\%$ & 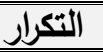 & سنوات الخبرة & s \\
\hline$\varepsilon 1.7 \mathrm{~V}$ & 0. & آقل من ه سنوات & 1 \\
\hline Y7.7V & re & من ه سنه إلى أقل من • اسنوات & $r$ \\
\hline$r \cdot . \wedge r$ & ro & من · ( سنوات إلى أقل من 10 سنه & $r$ \\
\hline $1 Y .0$. & 10 & من 10 سنه فأكثر & $\varepsilon$ \\
\hline $1 \ldots$ & $1 \%$. & الإجمالى & \\
\hline
\end{tabular}

المصدر : إعداد الباحثة من بيانات استمارة الاستبيان

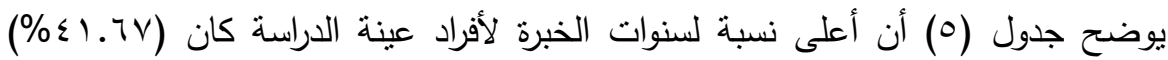

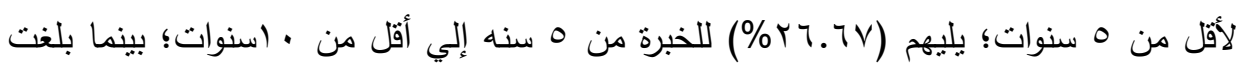

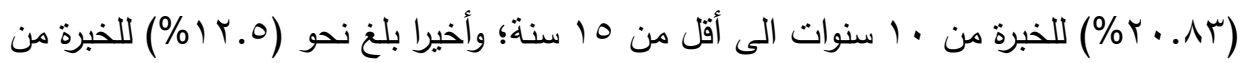
10 
أثر أهمية تطبيق عملية القياس والإفصاح المحاسبي عن التكاليف البيئية علي التنمية المستدامة بشركة الأرة بالعاشر من رمضان:

الفرض الأول: توجد علاقة ذات دلالة إحصائية بين القياس والإفصاح المحاسبي عن التكاليف البيئية في شركة الذرة بالعاشر من رمضان وأثره على رفع الوعي البيئي لاى المسئولين

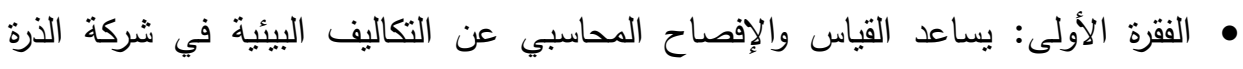

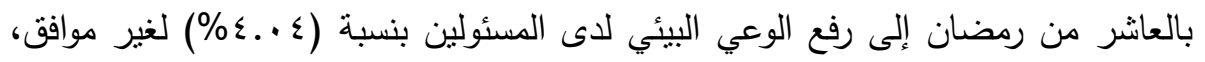

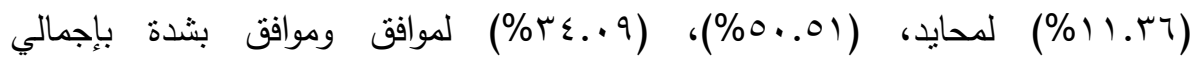
(\%^_.7.) المسئولين بشركة الذرة. الفقرة الثانية: بساعد القياس والإفصاح عن التكاليف البيئية في شركة الذرة بالعاشر من رمضان إلى غرس الوعي البيئي لاى الأطراف الأخرى كالموردين والبنوك والعملاء

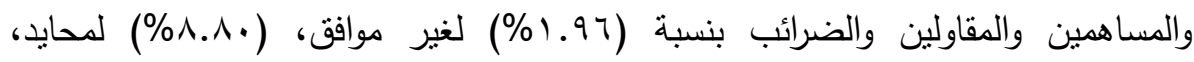

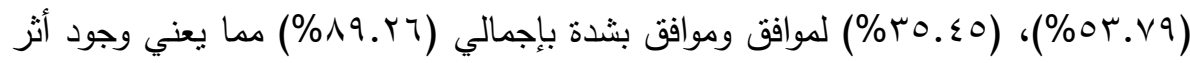
للقياس والإفصاح عن التكاليف البيئية علي غرس الوعي البيئي لدى الأطراف الأخرى كالموردين والبنوك والعملاء والمساهمين والمقاولين.

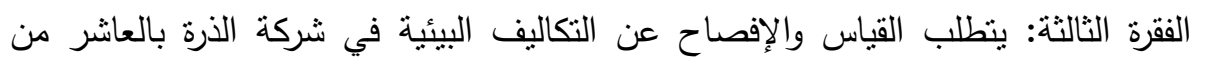

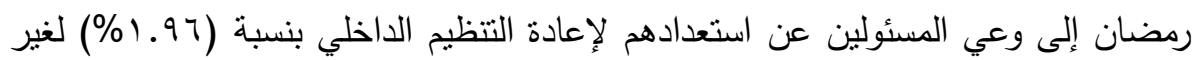

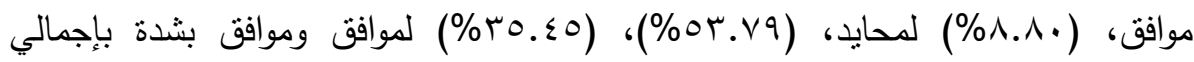

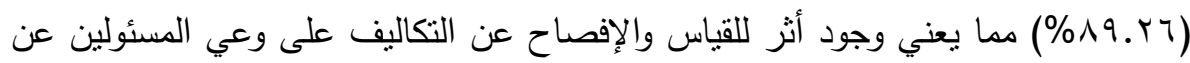
استعدادهم لإعادة التتظيم الداخلي. الفقرة الرابعة: بساعد القياس والإفصاح عن التكاليف البيئية في شركة الذرة بالعاشر من

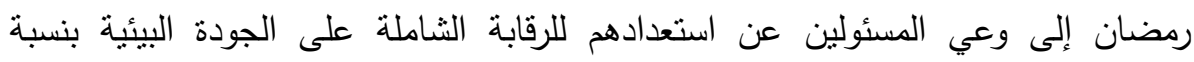

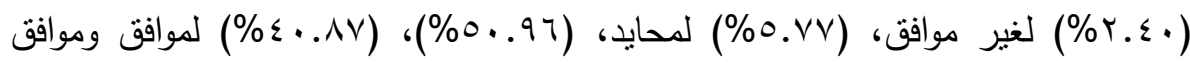

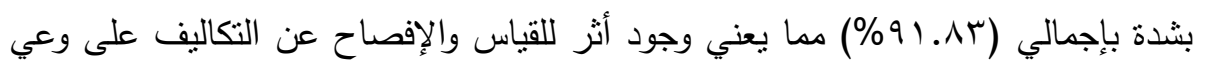
المسئولين عن استعدادهم للرقابة الثاملة على الجودة البيئية. 
الفقرة الخامسة: يتطلب القياس والإفصاح عن التكاليف البيئية في شركة الذرة بالعاشر من رمضان إلى وعي وتدريب العمال ليتمتعوا بمهارات بيئية متعددة تمكنهم بنسبة (به.o٪\%)

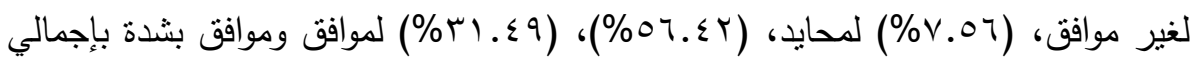
(\%^v.91)

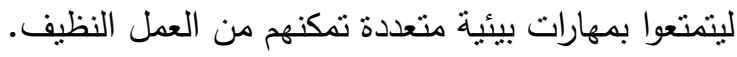
الفقرة السادة: يتطلب القياس والإفصاح عن التكاليف البيئية في شركة الذرة بالعاشر من

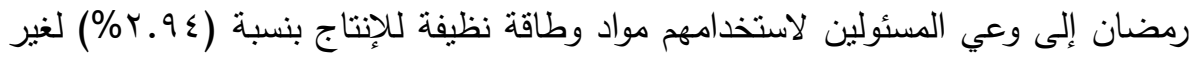

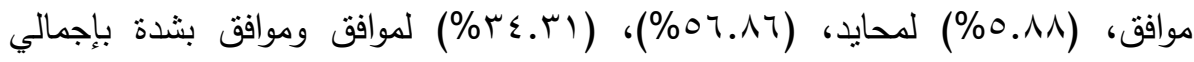
(\% ما يعني وجود أثز للقياس والإفصاح عن التكاليف على وعي المسئولين لاستخدامهم مواد وطاقة نظيفة للإنتاج. الفقرة السابعة: يتطلب القياس والإفصاح عن التكاليف البيئية في شركة الذرة بالعانشر من

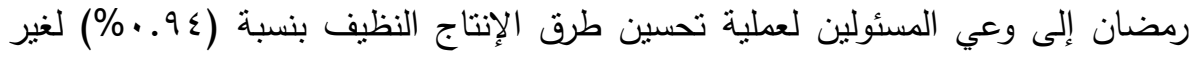

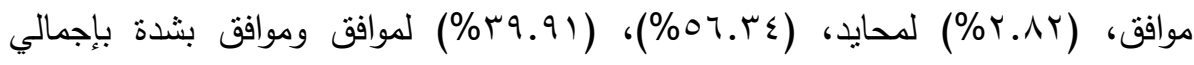

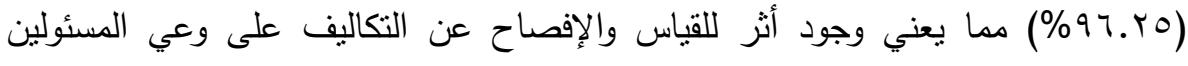
لعملية تحسين طرق الإنتاج النظيف. الفقرة الثامنة: يتطلب القياس والإفصاح عن التكاليف البيئية إلى وعي أخلاقي للمسئولين في

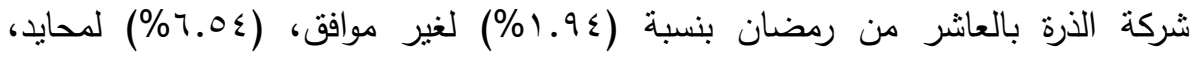

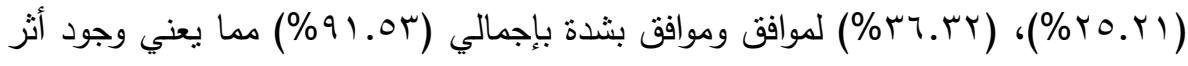
للقياس والإفصاح عن التكاليف على وعي أخلاقي للمسئولين في شركة الذرة بالعاشر من رمضان. • الفقرة التاسعة: يتطلب القياس والإفصاح عن التكاليف البيئية إلى وعي المسئولين للمنافسة

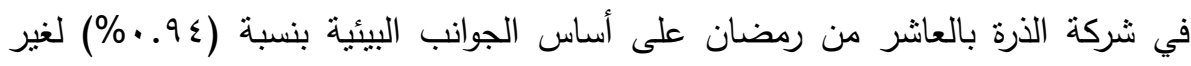

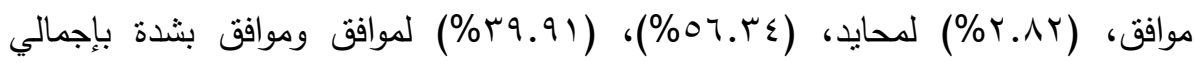




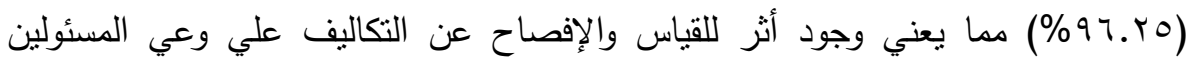
اللمنافسة في شركة الذرة بالعانشر من رمضان على أساس الجوانب البيئية.

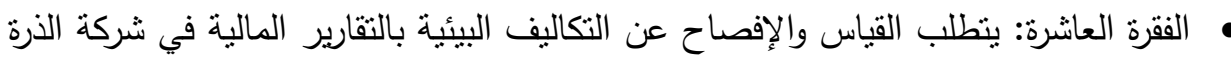

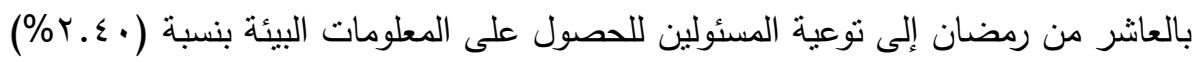

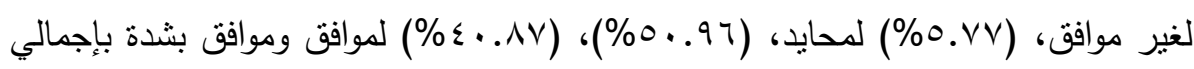
(r... (\%\%) مما يعني وجود أثز للقياس والإفصاح عن التكاليف علي توعية المسئولين للحصول على المعلومات البيئة. جدول(†): أثر أهمية تطبيق عملية القياس والإفصاح المحاسبي عن التكاليف البيئية على

\begin{tabular}{|c|c|c|c|c|c|c|c|c|c|c|}
\hline$\%$ & موافق & $\%$ & موافق & $\%$ & محايد & $\%$ & موافّت & $\%$ & بثير & الققرة \\
\hline rE..q & ס 1 & $0 . .01$ & $r \ldots$ & $11 . r 4$ & $\leqslant 0$ & $\varepsilon \ldots \varepsilon$ & 17 & . & . & 1 \\
\hline ro.కo & $1 \leq 0$ & or.vq & rY. & ᄉ.^. & ru & 1.97 & $\Lambda$ & . & . & r \\
\hline$\Gamma \varepsilon . \varepsilon \wedge$ & $1 \varepsilon$. & $1 V .00$ & YYE & rq.v & $r$. & $r .97$ & $1 T$ & . & . & $r$ \\
\hline$\wedge \vee . \Sigma$. & $1 V$. & 97.0. & 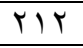 & $V V .0$ & $r \varepsilon$ & T.E. & 1. & $\cdot$ & . & $\varepsilon$ \\
\hline ¿q.r) & 1 ro & $\{Y .07$ & TrE & $07 . V$ & $r$. & $\varepsilon .01$ & 11 & • & • & 0 \\
\hline r. & $1 \varepsilon$. & 17.07 & YTr & $\wedge \wedge .0$ & Y $\varepsilon$ & Y. $9 \varepsilon$ & Ir & - & . & 7 \\
\hline (9.9) & IV. & 07.TE & $r \varepsilon$. & r.AY & $1 T$ & $.9 \varepsilon$ & $\varepsilon$ & - & - & V \\
\hline Tr.ru & 10. & Y1.00 & TrA & $0 \leqslant .7$ & YV & $1.9 \leq$ & $\Lambda$ & . & . & $\wedge$ \\
\hline$r 9.91$ & $1 V$. & $07 . \Gamma \leqslant$ & $r \leq$. & r.AY & Ir & $.9 \varepsilon$ & $\varepsilon$ & . & . & 9 \\
\hline AV.乏. & 18. & 97.0. & $r / r$ & $V V .0$ & TE & Y.E. & 1. & . & - & 1. \\
\hline
\end{tabular}

المصدر: إعداد الباحثة من بيانات استمارة الاستنيان

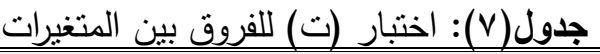

\begin{tabular}{|c|c|c|}
\hline \multicolumn{3}{|c|}{ اختبار (ت) للفروق بين المتغيرات } \\
\hline محايد & 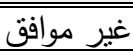 & \\
\hline$\Sigma \vee . T$ & $0 . . r$ & المتوسط \\
\hline $7 . .7$ & rᄉ. & التباين \\
\hline 1. & 1. & عدد المشاهدات \\
\hline & $99 . \%$ & Pooled Variance \\
\hline & . & معامل الاختلاف \\
\hline & 11 & Df \\
\hline & $\varepsilon \varepsilon . \varepsilon-$ & قيمة t t \\
\hline
\end{tabular}

جمعت وحسبت من بيانات استمارة الاستبيان باستخدام اختبار ت برنامج SPSS 
جدول(^): اختبار (ت) للفروق بين المتغيرات (ت) لمروف

\begin{tabular}{|c|c|c|}
\hline \multicolumn{3}{|c|}{ اختبار (ت) للفروق بين المتغيرات } \\
\hline موافق & 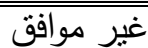 & \\
\hline r..0 & $0.1 . r$ & المتوسط \\
\hline$\varepsilon r . \uparrow$ & r^.l & التثاين \\
\hline $1 \cdot$ & 1. & عدد المشاهدات \\
\hline & $91 . r$ & الفرق المجمع \\
\hline & . & معامل الاختلاف \\
\hline & 11 & Df \\
\hline & $00.01-$ & 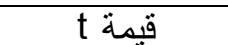 \\
\hline
\end{tabular}

جمعت وحسبت من بيانات استمارة الاستيان باستخدام اختبار ت برنامج SPSS جدول(9): اختبار (ت) للفروق بين المتغيرات اختبار (ت) للفروق بين المتغيرات

\begin{tabular}{|c|c|c|}
\hline \multicolumn{3}{|c|}{ اختبار (ت) للفروق بين المتغيرات } \\
\hline غير موافق & موافق بشدة & \\
\hline $0 . V . r$ & VV.MY & المتوسط \\
\hline rA.l & rA.1I & التباين \\
\hline 1. & 1. & عدد المشاهدات \\
\hline & Tr.T & الفرق المجمع \\
\hline & - & معامل الاختلاف \\
\hline & 11 & Df \\
\hline & $\leqslant 0 . \Gamma$. & قيمة t \\
\hline
\end{tabular}

جمعت وحسبت من بيانات استمارة الاستنيان باستخدام اختبار ت برنامج SPSS

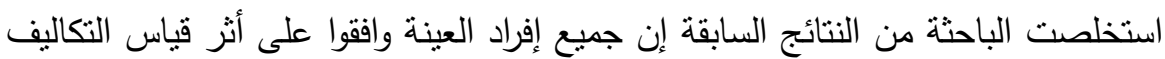
البيئية والإفصاح المحاسبي وتأثنير ها في رفع الوعي لدي المسئولين بشركة الذرة والعاملين بها إنها

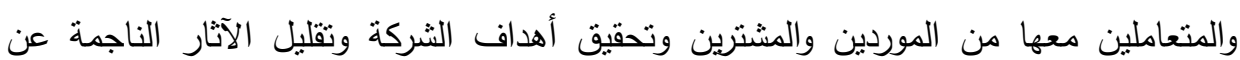

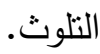
نتائج قياس أثر الفروض باستخدام التقدير الكمي للمتغيرات: نم الحصول علي

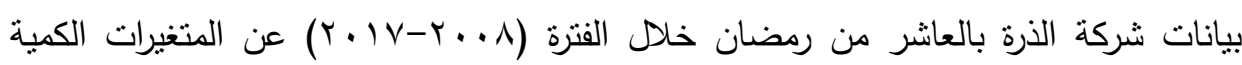
المرتبطة بالدراسة. الفرض الأول: "توجد علاقة طردية ذات دلالة إحصائية بين التكاليف البيئية وتكاليف تشغيل المنشأة ومبيعات الثركة". بدراسة العلاقة الانحدارية بين تكاليف تشغيل المنشأة والتكاليف البيأية دائية 
وإجمالي المبيعات لمنتجات الثركة بشركة الذرة بالعاشر من رمضان علاقة ارتباط موجبة

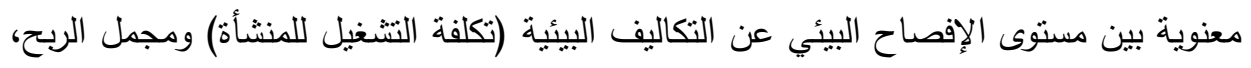

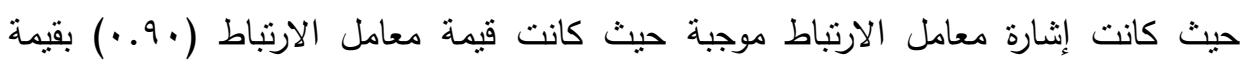

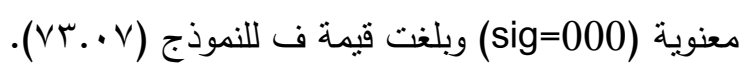
وقد أمكن صياغة العلاقة للنموذج على النحو التالي: $Y=66.48+0.257 \times 1$ $(2.42)^{* *}(8.55)^{* *}$ حيث أن، Y تعبر عن مجمل الربح بشركة الذرة تعبر عن المتغير التابع

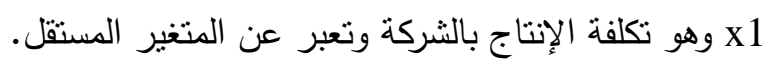
الفرض الثاني: "توجد علاقة طردية ذات دلالة إحصائية بين التكاليف البيئية ومستوى جودة بلتئية المنتج النهائية". بدراسة العلاقة الانحدارية بين للتكاليف البيئية وزيادة ربحية المنشاة بشركة الذرة بالعاشر من رمضان توجد علاقة ارتباط موجبة معنوية بين مستوى الإفصاح البيئي عن

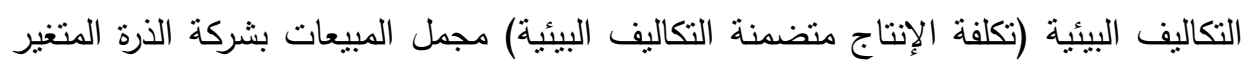

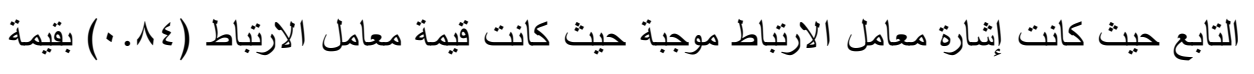

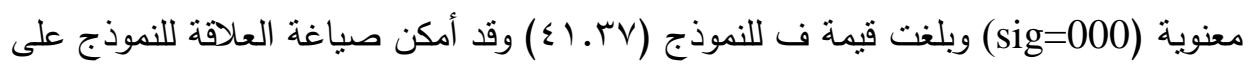
النحو التالي: $\mathrm{Y}=24.13+1.003 \times 1$ (0.813) (6.43)**

حيث أن، Y نعبر عن عن تكلفة تشغيل الإنتاج بالثركة المتغير التنابع x1 الفرض الثالث: "توجد علاقة طردية ذات دلالة إحصائية بين التكاليف البيئية تكلفة ضمن

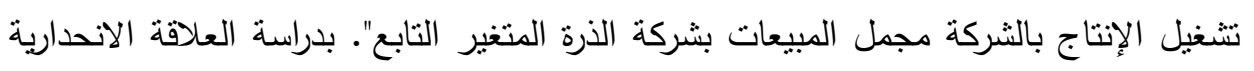

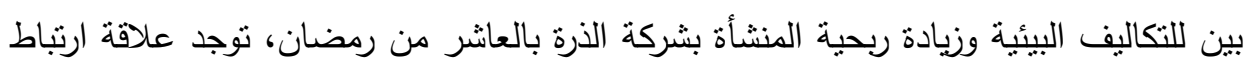

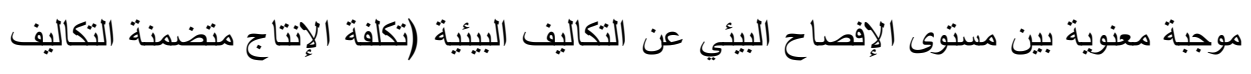

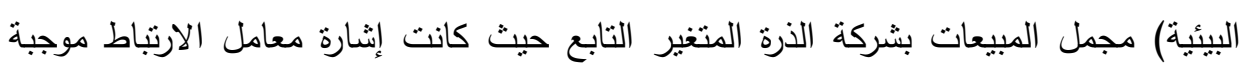


حيث كانت قيمة معامل الارتباط (عـ..) بقيمة معنوية (sig=000) وبلغت قيمة ف للنموذج

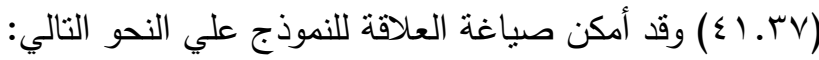
$\mathrm{Y}=148.4+1.003 \mathrm{x} 1$

$(3.70)^{* *}(19.3)^{* *}$ حيث أن Y تعبر عن مجمل المبيعات بشركة الذرة المتغير التابع x1 نعبر عن تكلفة تشغيل الإنتاج بالثركة جلول( • (1): مصفوفة الارتباط بين المتغيرات الاقتصادية والمالية للشركة الوطنية للذرة بالعاشر من رمضان

\begin{tabular}{|c|c|c|c|c|c|c|c|c|c|c|c|c|}
\hline لافيح قبلي & 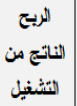 & المستزمات & تكلفة الانتاج & $\begin{array}{l}\text { المبيعات من } \\
\text { المنتيسية } \\
\text { المئيسة }\end{array}$ & المبيعات من المنتجات & العاملين & لريحي & قأقيدة & تكلفة & جبيعات & لربيح & \\
\hline & & & & & & & & & & & $\ldots .1$ & مجل الربح \\
\hline & & & & & & & & & & $\cdots 1$ & १६१.. & جملة المبيعات \\
\hline & & & & & & & & & $\ldots .1$ & १८१.. & $910 .$. & نكالفة الإنثاج \\
\hline & & & & & & & & $\cdots 1$ & qזध.. & QYY.. & ArE.. & قَيمة الأجور \\
\hline & & & & & & & $\cdots .1$ & Doß. & . & 100. & vVY.. & صافي الربح \\
\hline & & & & & & $\cdots 1$ & זrז. & orq.. & rer.e. & riv.e & $111 .$. & عدد العاملين \\
\hline & & & & & $\cdots .1$ & orr.e & rV£.. & $9+\wedge$. & $\wedge 9 . .$. & Nitr. & $79 \leqslant .$. & المنئجات الثنانوية من \\
\hline & & & & $\cdots .1$ & $907 .$. & ira." & $\{r \mu$. & १६४. & AqY. & 110. & קוז. & المنتجات الرئيسبية من \\
\hline & & & $\cdots .1$ & ^९Y.. & $\wedge 9 . .$. & rer.e. & . & qזะ. & $\ldots .1$ & 9८9.. & 910.0 & نكلفة الإنثاج \\
\hline & & $\cdots, 1$ & १११.. & MAr. & AYY. & $r \cdot \varepsilon_{.}$. & Tr. & ११५. & १११.. & $9 \wedge \wedge$. & ११४. & نكلفة المستلزمات \\
\hline & $\cdots 1$ & $\varepsilon 1 \Lambda_{.}$ & \& १.. & $\{10 .$. & mo.e. & ه१५.. & $v r v .$. & $\varepsilon \wedge \varepsilon .$. & ¿rq.. & ६१४.. & ०१Y. & الربح الثائج من \\
\hline$\cdots \cdot 1$ & $v_{1} \ldots$. & 701. & $709 .$. & $010 .$. & $01 \varepsilon .$. & $00 .$. & q.V.. & Tro.. & 709.. & $T \& \Upsilon_{.} \cdot$ & TAV. . & صافي الربح قبل \\
\hline
\end{tabular}

توضح مصفوفة الارتباط وجود ارتباط موجب بين المتغيرات التي تم بناء العلاقات

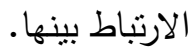




\section{التيوكيانش}

فى ضوء النتائج التي تم التوصل إليها في كل من الدراسة النظرية والتطبيقية توصي الباحثة بما

$$
\text { يلي: }
$$

1-زيادة الوعي المؤسسي والجمعيات الأهلية فى البيئة المصرية بأهمية الإفصاح المحاسبي عن البهن

التكاليف البيئية بالثركات الصناعية من خلال منظومة الوعي البيئي لتحقيق الإفصاح

$$
\text { المحاسبي والبيئي. }
$$

r-الحاجة إلى إصدار معيار محاسبي من قبل الجهات والمنظمات المهنية لتنظيم عملية الإفصاح المحاسبي والبيئي عن النكاليف البيئية، مما يؤدى إلى زيادة جودة المعلومات

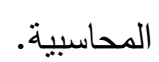

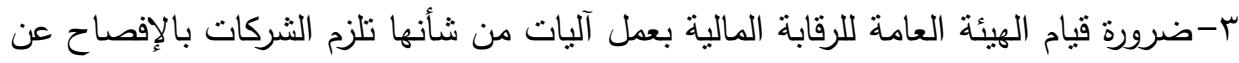

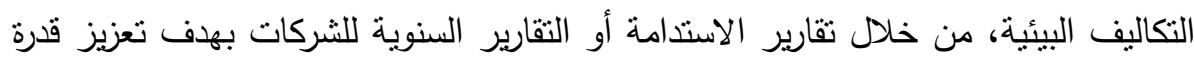

المستثمرين على إدراك الفرص الاستثمارية البديلة، ومن ثم اتخاذ القرارات السليمة.

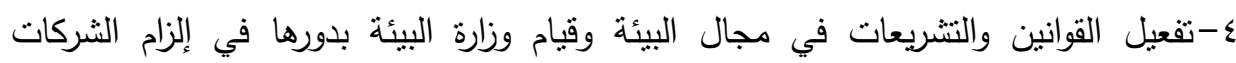
بضرورة العمل على خفض أثار النكاليف البيئة وترشيد استخدام الطاقة.

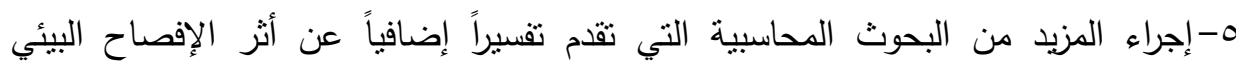
والمحاسبي عن النكاليف البيئية فى ظل الدتغيرات الثقافية والدجتمعية في البيئة الدصرية.

\section{extl}

ناظم شعبان جبار، واياد: تحميل البيانات المحاسبية عن الأداء البيئي وأثره في حماية البيئة

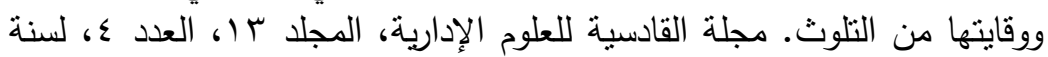

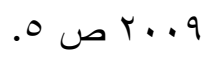

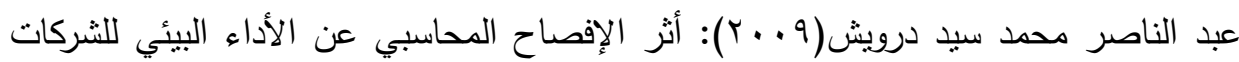

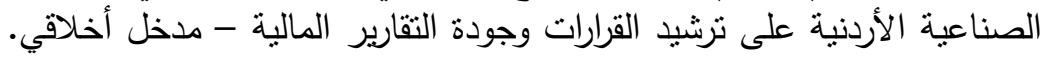

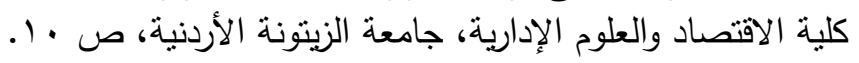




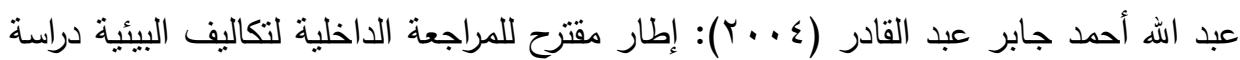

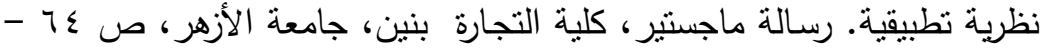

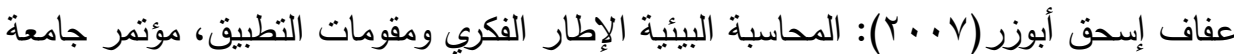

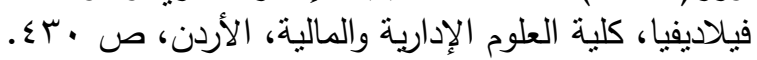

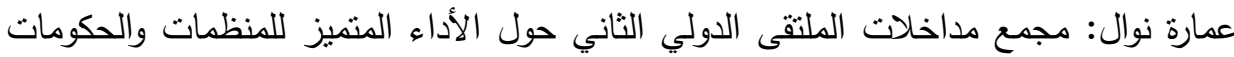

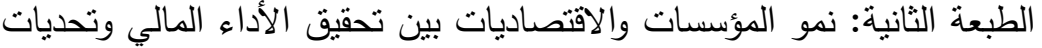

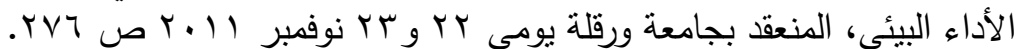

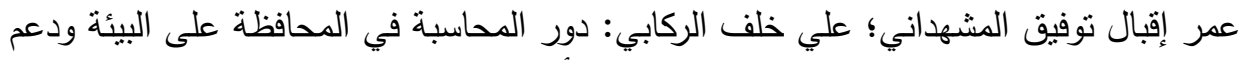

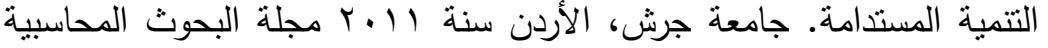

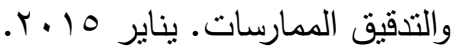

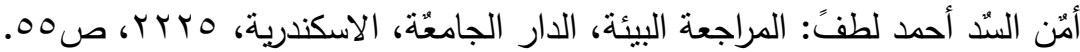

لطيف زيود وآخرون: دور الإفصاح المحاسب، فه، سوق الأوراق المالية فه، ترشيد قرار

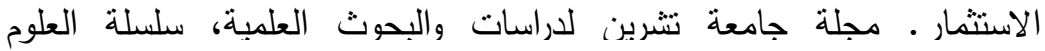

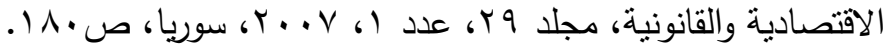

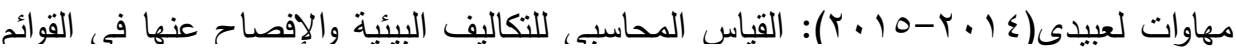

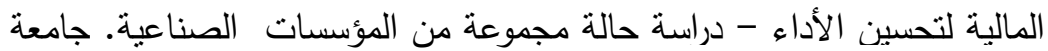

محمد خضير بسكرة، الجزائر •

همت مصطفى هندي(997 (197): أثز العوامل البيئية على تحديد سعر الفائدة في البنوك المصرية.

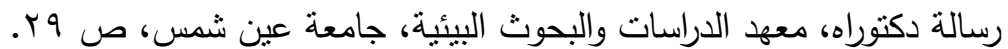

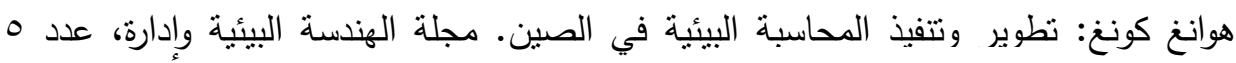

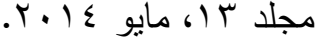

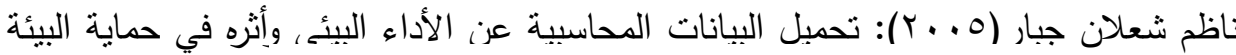

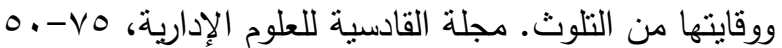

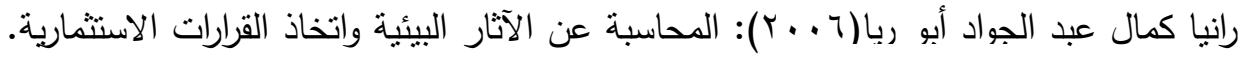
رسالة ماجستير •

عبد الله بن جمعان الغامدى(V . . r): التتمية المستدامة بين الحق في استغلال الموارد الطبيعية والمسئولية عن حماية البيئة.

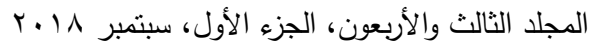


عبد الهادى الرفاعي،، باسل أسعد: التلوث البيئ، الناجم عن الصناعة الثقيلة إمكانية قياسه

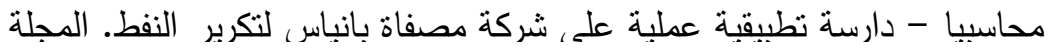

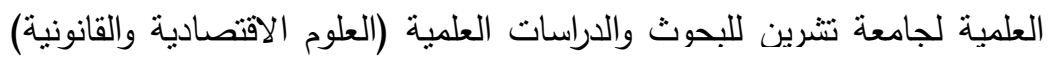

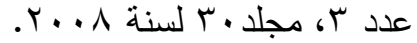

هدى سراج الدين محمد(1) (1): التكاليف البيئية ودورها في تقويم الأداء البيئي في المنشآت الصناعية. رسالة ماجسنير

Ahmed, M. (2002): Theoretical Framework for Environmental Accounting: Application on the Egypt Petroleum Sector. Vol: 55, No: 30, pp. 420, 421, Accounting Journal.

Çelik, M. (2008): İşletmeler Açısından Çevresel Maliyetler, Çevre Finansmanı Muhasebe ve Finansman İçin Yeni Trendler, Gazi Kitabevi, Editör: Ayrıçay Y. \& Karataş, A. 1.Bask1, Ankara.

Halftrack, V. and Soylu, K. (2008): Çevresel Belligerent Muhasebesi ve Raporlanmas1, Kocaeli Universities Sosyal Bilimler Dergisi, Say1:15, 1:92-113

Kırımhan, S. (2005): Caver Yönetimi: Nüfus, Kaynak ve Carve İlişkileri, Ankara.

\title{
THE IMPACT OF ENVIRONMENTAL COST ACCOUNTING TO MAKE DECISIONS TO ACHIEVE SUSTAINABLE DEVELOPMENT
}

El-Sayed, Henda, M. ${ }^{(1)}$; Amer, A. H. ${ }^{(2)}$ and Ahmed, R. A. ${ }^{(3)}$ 1) Arabic Will for Trading and Industries 2) Faculty of Commerce, Ain Shams University 3) Faculty of Science, Mansoura University

\begin{abstract}
The study aimed at explaining the impact of environmental cost accounting and disclosure on decision making for sustainable development, and presenting proposals for its development. (100)


questionnaires were analyzed and (100) questionnaires were used. T test was used for each sample to test the hypotheses of the study. The researchers found that there is a statistically significant relationship between the environmental costs and their disclosure, and the development of awareness among officials, managers and employees, which is reflected in the impact of achieving sustainable development, and showing a statistically significant relationship between, and it was found that there is no application of environmental costs and disclosure of environmental costs and disclosure and the extent of their environmental application. 
The study suggested some recommendations to develop the application of accounting for the lack of qualified human cadres, and finally on the environmental costs and disclosure to provide information at the company on the tenth of Ramadan, the study recommends the need to be applied Cost accounting and environmental disclosure procedures help to make good decisions. With an accounting system in the maize company that provides a lot of information to be disclosed in the financial statements, the study recommends that the cost accounting and environmental disclosure procedures in the company should be applied so as to improve the fairness of the financial statements, And the financial section of the importance of the application of costs and environmental disclosure of the industrial company of corn, and it also recommends the need for training modules in environmental accounting and the need for the adequacy of staff working in the accounting department who are fully familiar with environmental accounting. 